\title{
Die organisierte Vermarktlichung der Moral und die Moralisierung der Märkte. Eine vergleichende Analyse der Fair-Handelsbewegung und der Entstehung ethischen Konsums in Europa
}

\author{
Sebastian Koos
}

Zusammenfassung Die Studie untersucht am Beispiel der Fair-Handelsbewegung, wie sich die Entstehung und Verbreitung des ethischen Konsums in Europa seit dem Ende der 1990er-Jahre erklären lässt. In Ergänzung zu den existierenden akteurszentrierten Erklärungen ethischen Konsums werden Ansätze sozialer Bewegungstheorien verwendet, welche die organisationalen Dimensionen der Fair-Handelsbewegung und die nationalen Opportunitätsstrukturen in den Blick nehmen. Im Artikel wird argumentiert, dass der Wandel der Feldlogik der Fair-Trade-Bewegung von einer zivilgesellschaftlichen hin zu einer Marktlogik einen zentralen Impuls für die Entstehung und die Diffusion des Konsums fair gehandelter Produkte, aber auch für das Wissen über Fair Trade darstellte. Die Hypothesen werden anhand einer Mehrebenen-Analyse mit Daten einer Eurobarometer-Umfrage (1997) überprüft, die um Informationen zu den nationalen Fair-Trade-Organisationen und den ökonomischen und kulturellen Opportunitätsstrukturen ergänzt wurden. Die Ergebnisse stützen die These des zentralen Einflusses der organisierten Vermarktlichung auf die Moralisierung der Märkte und weisen auf die spezifischen Mobilisierungsstrategien der FairHandelsbewegung hin.

Schlüsselwörter Ethischer Konsum · Fairer Handel · Institutionelle Logik · Soziale Bewegung $\cdot$ Europa $\cdot$ Diffusion 


\title{
The organized marketization of morality and the moralization of markets. A comparative analysis of the fair trade movement and the diffusion of ethical consumption in Europe
}

\begin{abstract}
This paper tries to show how the change in the organizational logic of the fair trade movement, embodied by fair trade labelling, has sparked the growth of ethical consumption. By establishing fair trade labels, the initial movement logic of political influence through education was supplemented and partly abandoned in favor of a market logic. Fair trade movements in Western Europe differ in the way they organize and market fair traded goods. Drawing on organizational institutionalism and social movement theories of economic opportunity structures, it is elaborated how the emergence of a new organizational form and its underlying logic shape consumption patterns. Hypotheses are empirically tested using a quantitative multilevel design. Organizational data on national fair trade movements compiled from an organizational survey of the European Fair Trade Association is combined with individual level survey data of the 1997 Eurobarometer for 12 European countries. Logistic hierarchical regression models reveal the crucial importance of the fair trade labels once diffused into consumer markets, controlling for organizational communication efforts as well as the number of distribution channels for individual fair trade consumption. Thus, adopting a market logic has been a powerful force in rendering fair trade successful.
\end{abstract}

Keywords Ethical consumption $\cdot$ Fair trade $\cdot$ Institutional logic $\cdot$ Social movements $\cdot$ Europe $\cdot$ Diffusion

\section{Le marketing organisé de la morale et la moralisation des marchés. Une analyse comparée du mouvement du commerce équitable et de l'émergence de la consommation éthique en Europe}

Résumé Cet article tente d'expliquer l'émergence et la diffusion de la consommation éthique en Europe depuis la fin des années 1990 en analysant l'exemple du commerce équitable. En complément des explications existantes de la consommation éthique centrées sur les acteurs, l'approche utilisée ici s'inspire des théories des mouvements sociaux et prend en compte les dimensions organisationnelles du mouvement du commerce équitable et les structures d'opportunités nationales. La thèse défendue est que le passage d'une logique citoyenne à une logique de marché comme modalité d'action du mouvement du commerce équitable a donné une impulsion décisive à l'émergence et à la diffusion de la consommation de produits équitables ainsi qu'à la connaissance du commerce équitable. Ces hypothèses sont vérifiées à l'aide d'une analyse multi-niveaux conduite à partir de données d'un sondage Eurobaromètre (1997) complétées par des informations sur les organisations nationales du commerce équitable ainsi que sur les structures d'opportunités économiques et culturelles. Les résultats étayent la thèse d'une influence décisive du marketing organisé sur la moralisation des marchés et mettent en évidence les stratégies de mobilisation spécifiques du mouvement du commerce équitable. 
Mots-clés Consommation éthique · Commerce équitable $\cdot$ Logique institutionnelle $\cdot$ Mouvement social $\cdot$ Europe $\cdot$ Diffusion

\section{Einleitung}

Die Fair-Handels- bzw. Fair-Trade-Bewegung ${ }^{1}$ ist eine transnationale soziale Bewegung, die bestrebt ist, die Probleme des globalen Welthandels aufzuzeigen und einen alternativen fairen Handel zu etablieren (FLO 2005; Krier 2006, S. 21). Dazu werden Produkte aus dem globalen Süden mit einem Preisaufschlag versehen, der den Produzenten die Existenzgrundlage sichern, Armut verringern und zu einer nachhaltigen wirtschaftlichen und politischen Entwicklung beitragen soll. Für die fair gehandelten Güter, wie beispielsweise Kaffee, Tee, aber auch Fußbälle und Blumen, erhalten die Produzenten einen garantierten Mindestpreis, der über dem Weltmarktpreis liegt. Der Preis wird von der Dachorganisation Fairtrade International (FLO) unter Mitwirkung von verschiedenen Anspruchsgruppen festgelegt (FLO 2005). Ein Teil der Erlöse muss von den Produzenten direkt in Infrastrukturmaßnahmen, wie z. B. in Schulen, investiert werden, um die Grundlage für eine nachhaltige Entwicklung zu schaffen. Neben diesen entwicklungspolitischen Zielen verfolgt die FairHandelsbewegung aber auch das Ziel, die Bürger des globalen Nordens über die Probleme des Welthandels aufzuklären und politische Lösungen zur Änderung des globalen Handelssystems anzustoßen (Krier 2006, S. 21).

Der Faire Handel wurde in den 1960er-Jahren in den Niederlanden und Großbritannien begründet und zumeist von religiös orientierten „Dritte Welt“-Gruppen geleitet, die neben dem Verkauf von Gütern das Ziel verfolgten, über ungerechte Handelsbedingungen zu informieren. Seit Ende der 1990er-Jahre hat sich die Bewegung stärker auf die Organisation eines alternativen Welthandels konzentriert und erzielt mittlerweile beachtliche Umsätze bei kontinuierlichen Wachstumsraten. Dabei leistet der Faire Handel einen Beitrag zur Verbesserung der Lebensbedingungen von Produzenten in Süd- und Mittelamerika, in Afrika und Asien (FLO 2010; Krier 2007; Nicholls und Opal 2005).

Während ,traditionelle“ soziale Bewegungen, wie z.B. die Arbeiterbewegung, heute große Probleme bei der Gewinnung und Mobilisierung von Mitgliedern haben (Ebbinghaus und Visser 1999), ist die „Erfolgsgeschichte“ (Krier 2007) des Fairen Handels überraschend. Sie steht beispielhaft für die Entstehung und Diffusion des ethischen Konsums in modernen Industrie- und Dienstleistungsgesellschaften. Ethischer Konsum meint dabei, dass bestimmte ethische Prinzipien, wie z. B. das der Achtung von Menschenrechten oder das der ökologischen Nachhaltigkeit, leitend bei der Entscheidung für oder gegen ein Produkt sind. Ein derartig motivierter Konsum hat das Ziel, als verwerflich wahrgenommene politische und ökonomische Praktiken und Institutionen zu verändern (Micheletti 2003, S. 2). Die zunehmende Verbreitung ethischer Konsummuster lässt sich als eine „Moralisierung der Märkte“

\footnotetext{
1 Fairer Handel und Fair Trade werden nachfolgend synonym verwendet. Für Fairer Handel wurde die Großschreibung gewählt, weil und insofern es um die institutionalisierte Form dieser konsumethischen Bewegung geht, also nicht nur um eine Form des fairen Verhaltens im weiten Sinne.
} 
bezeichnen (Stehr 2007). Wie lassen sich die Entstehung des ethischen Konsums und die damit einhergehende Moralisierung der Märkte erklären?

Ein Großteil der Forschung zum ethischen Konsum bzw. zum Kauf von fair gehandelten Produkten widmet sich akteurszentrierten Erklärungen, die häufig organisationale und institutionelle Prozesse sowie den strukturellen Kontext, in welchem das Kaufverhalten eingebettet ist, schon als gegeben voraussetzen (Arndorfer und Liebe 2013; Pelsmacker und Janssens 2007; Shaw et al. 2005; Sunderer und Rössel 2012). Während die Berücksichtigung der individuellen Motivlagen und Ressourcen notwendig ist, um das Kaufverhalten des Einzelnen zu verstehen, scheinen die institutionellen Prozesse auf der Meso- und Makroebene gleichfalls wichtig, um die Verbreitung des ethischen Konsums als gesellschaftliches Phänomen zu erklären (Bartley et al. 2015; Micheletti und Stolle 2012). Aus diesem Grund werden in diesem Beitrag organisationstheoretische Ansätze sowie Theorien aus der sozialen Bewegungsforschung zur Erklärung der „Mobilisierung“ für ethischen Konsum und der Verbreitung des Wissens um Fair Trade herangezogen.

Das Hauptargument dieses Aufsatzes ist, dass eine seit den 1990er-Jahren einsetzende Feldlogik der Vermarktlichung für das enorme Wachstum des Fairen Handels und die Entstehung von ethischem Konsum zentral war. Die Einführung von Produktsiegeln spiegelt dabei einen Wandel der Feldlogik der Fair-Handelsbewegung wider, in dem eine neue Marktlogik im Sinne eines ,institutional layering“ (Thelen 2004) die traditionelle Gemeinschaftslogik des Fairen Handels überlagert, aber nicht vollkommen ersetzt. Der Prozess der Setzung bestimmter sozialer oder ökologischer Produktionsstandards sowie deren Zertifizierung und deren Sichtbarmachung durch Produktlabel lassen sich als Kommodifizierung normativer Ansprüche verstehen. Dieser Prozess führt zur Institutionalisierung von ethischen Kriterien auf Märkten und befördert die Ausbreitung von ethischem Konsum. Alternative Erklärungen nehmen die Mobilisierungsstrukturen, Framing-Strategien sowie ökonomische und kulturelle Opportunitätsstrukturen des Fairen Handels in den Blick, um die unterschiedliche Verbreitung des ethischen Konsums und des Wissens um den Fairen Handel in Europa zu erklären.

Im nachfolgenden Abschnitt wird zunächst im Anschluss an die Konzepte der institutionellen Logik und „field frames“ die Idee der organisierten Vermarktlichung und der Einfluss dieser Vermarktlichung auf den Kauf von Fair-Trade-Produkten vorgestellt (Davis et al. 2005; Lounsbury et al. 2003; Scott 2008; Thornton und Ocasio 2008). Danach werden klassische (Meso-Ebenen-)Ansätze der Mobilisierungsforschung diskutiert und auf den Konsum fair gehandelter Produkte übertragen. Nach einer knappen Diskussion von individuellen Determinanten des Konsums von FairTrade-Gütern werden die eigenen Hypothesen vorgestellt und mit vergleichenden empirischen Analysen überprüft. Als Analysezeitpunkt wurden die 1990er-Jahre gewählt, da hier noch starke Länder-Unterschiede hinsichtlich der Einführung eines Fair-Trade-Siegels und dessen organisationaler Institutionalisierung bestehen. In der Untersuchung werden Daten einer Eurobarometer-Umfrage von 1997 mit Organisationsdaten der Fair-Handelsbewegungen in Europa verknüpft. Es zeigt sich, dass die organisierte Vermarktlichung einen statistisch robusten Einfluss auf den Konsum fair gehandelter Produkte hat. Abschließend wird die Entwicklung des FairTrade-Konsums von 2001 bis 2007 im deutsch-britischen Vergleich skizziert, um 
das weitere Wachstum des Fairen Handels zu illustrieren. Das Beispiel der FairHandelsbewegung zeigt, wie eine soziale Bewegung den Markt selbst als Plattform für eine Kritik am Markt und seiner Funktionsweise nutzen kann.

\section{Theoretischer Hintergrund}

In der sozialen Bewegungsliteratur werden mindestens drei Ansätze zur Erklärung der Mobilisierung für kollektives Handeln unterschieden (McAdam et al. 1996b). Der erste Ansatz verweist auf die politischen Opportunitätsstrukturen oder Umweltbedingungen für die Mobilisierung und den Erfolg der sozialen Bewegungen (Meyer 2004; Tarrow 1994). Die zweite Theorie konzentriert sich auf die informellen und formalen Strukturen, die Akteure zur Teilnahme an kollektivem Handeln bewegen (McCarthy 1996). Schließlich beschäftigt sich die dritte Gruppe von Erklärungen kollektiven Handelns mit sogenannten „Framing“-Bemühungen der Bewegungsaktivisten (Benford und Snow 2000; Snow und Benford 1988). Die drei Ansätze sind kaum klar abgrenzbar, überlappen sich und werden in verschiedenen Kombinationen verwendet, um den Erfolg von sozialen Bewegungen zu erklären (McAdam et al. 1996a).

In letzter Zeit ist ein wachsendes Interesse an der Kombination der Ansätze der sozialen Bewegungsforschung mit Organisationstheorien entstanden (Davis et al. 2005; Lounsbury 1997; Rao 1998; Rao et al. 2000). Beide Forschungsfelder teilen ein Interesse an der Organisation kollektiven Handelns, seien es die Koordination von Arbeit in einem Unternehmen oder die Teilnahme von Bürgern an Protest. Bürokratische Formen der Organisation sind nicht nur ein zentraler Bestandteil der betrieblichen Unternehmensführung, sondern spielen auch eine große Rolle bei der Steuerung und Verwaltung von Protestpolitik durch soziale Bewegungsorganisationen (McAdam und Scott 2005). Im Folgenden wird zunächst die sich verändernde institutionelle Feldlogik der Fair-Trade-Bewegung dargestellt und ihr Einfluss auf die Entstehung des ethischen Konsums skizziert, bevor der Einfluss von Mobilisierungsstrukturen und „Framing“-Aktivitäten diskutiert wird. Anschließend werden einige theoretische Überlegungen zur Bedeutung von wirtschaftlichen und kulturellen Gelegenheitsstrukturen dargestellt und schließlich kurz der Einfluss von Individualmerkmalen wie z. B. persönliche Ressourcen oder Motivationen dargestellt.

\subsection{Organisationale Triebkräfte fairen Konsums}

\subsubsection{Vermarktlichung als institutionelle Feldlogik im Fairen Handel}

Ein Ansatz zur Integration von sozialen Bewegungs- und organisationssoziologischen Theorien konzentriert sich auf die Rolle der institutionellen Logiken (Lounsbury et al. 2003; McAdam und Scott 2005). Institutionelle Logiken können verstanden werden als ,belief systems and associated practices that predominate in an organizational field“ (Scott et al. 2000, S. 170). McAdam und Scott (2005) verweisen darauf, dass das Konzept der institutionellen Logik große Ähnlichkeit mit dem „Framing“-Konzept aus der Bewegungsforschung aufweist, welches „, the collective 
processes of interpretation, attribution, and social construction that mediate between opportunity and action" bezeichnet (McAdam et al. 1996a, S. 2). Framing wird jedoch als bewusster Prozess oder Vorgang aufgefasst, in dem bestimmte Bedeutungen oder Interpretationen konstruiert werden, während unter institutionellen Logiken extern gegebene Skripte und Rationalitäten von organisatorischen Feldern verstanden werden. Aus diesem Grund schlagen Lounsbury et al. (2003) in Anlehnung an Pierre Bourdieu das Konzept der „field frames“ oder Feldlogiken vor, welches beinhaltet, dass diese Logiken durchaus „bewusst“ verändert werden können, jedoch gleichwohl die Handlungsrationalitäten in gesellschaftlichen und organisationalen Felder prägen.

Bestimmte Logiken sind immer auch mit bestimmten Organisationsformen verknüpft, die mehr oder minder geeignet erscheinen, um Probleme auf den jeweiligen organisationalen Feldern zu bearbeiten (Clemens 1996). Das heißt, bestimmte Organisationsformen sind inhärent mit Deutungen verknüpft, die Handlungsmuster zur Lösung bestimmter Probleme bereitstellen. Wenn sich Organisationsformen wandeln oder sich gar neue Formen ausbilden, verändern sich dadurch auch die Feldlogiken. Ganz allgemein können Organisationsformen hinsichtlich ihrer Ziele, Autoritätsbeziehungen, Technologien und Marketing-Strategien charakterisiert und unterschieden werden (Rao et al. 2000). Im Fairen Handel wurde in den späten 1980er-Jahren eine neue Organisationsform geschaffen, die sogenannten ,Labelling-Organisationen“, die Standards - wie z. B. gerechte Anbau- und Arbeitsbedingungen - festlegen und Produktsiegel für die Einhaltung der Standards vergeben (Renard 2003). Bis in die späten 1980er-Jahre war die Fair-Handelsbewegung durch lose gekoppelte Alternative Handelsorganisationen (ATO), Weltläden und kirchliche Gruppen gekennzeichnet. 1988 wurde dann in den Niederlanden unter der Leitung der „Fair Trade Organisatie“ die erste Labelling-Organisation gegründet, die das Siegel „Max Havelaar" für fair gehandelte Produkte vergab (Fridell 2004).

Diese Organisationsform verbreitete sich schnell über die benachbarten Länder, sodass innerhalb der nächsten zehn Jahre zehn weitere Labelling-Organisationen in anderen europäischen Ländern gegründet wurden. Die Zertifizierung eines Produktes entsprechend der Fair-Trade-Standards und seine Besiegelung durch diese Organisationen stellen ein sichtbares Signal über die normative Qualität eines Produktes bereit und führen so zu einer neuen ethischen Differenzierung auf Märkten (Renard 2003). In diesem Prozess werden die normativen Standards des Fairen Handels kommodifiziert, indem die ethische Qualität zum Teil der Ware selbst wird und so fair gehandelte Produkte für den Massenkonsum zugänglich gemacht werden (Fridell 2007). Durch die Einführung der Produktbesiegelung wurde die bis in die 1980er-Jahre dominierende zivilgesellschaftliche Koordinationslogik der Bewegung (Renard 2003, S. 89) durch eine Marktlogik ergänzt und teilweise ersetzt. Die Einführung von Produktsiegeln spiegelt einen Wandel der Feldlogik der FairHandelsbewegung wider, der darin besteht, dass eine neue Marktlogik im Sinne eines ,institutional layering“ (Thelen 2004) die traditionelle Gemeinschaftslogik des Fairen Handels überlagert, aber nicht vollkommen ersetzt. Dies kann als ein Prozess der organisierten „Vermarktlichung“ von ethisch fairen Tauschbeziehungen verstanden werden. Gleichzeitig wurde die bestehende Marktlogik auf den Märkten des 


\begin{tabular}{|l|l|l|}
\hline & $\begin{array}{l}\text { Fair-Trade-Bewegung } \\
\text { 1960er- bis 1980er-Jahre }\end{array}$ & $\begin{array}{l}\text { Fair-Trade-Bewegung } \\
\text { 1990er-Jahre bis heute }\end{array}$ \\
\hline \multicolumn{1}{|c|}{ Dominierende Logik } & $\begin{array}{l}\text { Zivilgesellschaftliche } \\
\text { Koordinationslogik } \\
\text { der Organisationsformen }\end{array}$ & Markt-Logik \\
\hline Ziele & $\begin{array}{l}\text { Netzwerk-Durchdringung, } \\
\text { ausreichender Umsatz, } \\
\text { Bewusstseinsbildung }\end{array}$ & $\begin{array}{l}\text { Markt-Durchdringung, } \\
\text { hoher Umsatz, } \\
\text { Mainstreaming }\end{array}$ \\
\hline Autoritätsbeziehungen & Flache Hierarchie & $\begin{array}{l}\text { Spezialisierte, hierarchisch } \\
\text { geordnete Stellen }\end{array}$ \\
\hline Technologien & $\begin{array}{l}\text { Zivilgesellschaftliche } \\
\text { Netzwerke }\end{array}$ & Zertifizierung \\
\hline Marketing-Strategien & $\begin{array}{l}\text { Anhänger, Kirchenmitglieder, } \\
\text { Nischenprodukte, } \\
\text { niedrige Qualität }\end{array}$ & $\begin{array}{l}\text { Konsumenten, } \\
\text { Massenkonsumgüter, } \\
\text { mittlere bis hohe Qualität }\end{array}$ \\
\hline
\end{tabular}

Abb. 1 Der Wandel der Feldlogik der Fair-Handels-Bewegung

Massenkonsums durch eine sichtbar gemachte Morallogik ergänzt. Dies wiederum trägt zu einer „Moralisierung“ der Konsummärkte bei.

Ganz allgemein bezeichnet „Vermarktlichung“ einen Vorgang, bei dem Güter, welche zuvor nicht zum Verkauf standen, mit einem bestimmten Preis versehen werden. Diese Güter werden so für den Austausch zwischen Verkäufern und Käufern verfügbar, wobei sich der Preis aus Angebot und Nachfrage ergibt (Ertman und Williams 2005, S. 1 f.). Dieser Prozess wird im Fall des Fairen Handels organisiert, was bedeutet, dass die Preisfindung nicht spontan aus einer Interaktion zwischen wirtschaftlichen Akteuren erwächst, sondern planvoll und kontrolliert abläuft und von einer neuen Organisationsform (der Labelling-Organisation) verwaltet wird. Organisierte Vermarktlichung ethischer Tauschbeziehungen meint damit nicht nur, dass beispielsweise Kaffee von kleinen Kooperativen in Lateinamerika in westlichen Supermarktketten identifizierbar und verfügbar geworden ist, sondern auch, dass die Fair-Trade-Standards, die durch ein Fair-Trade-Label signalisiert werden, als besondere Qualität eines Produktes der Dynamik der Marktkräfte zugänglich werden. Mit Marktkräften ist dabei zum einen der Wettbewerb zwischen verschiedenen Anbietern (Herstellern, Supermarkt- oder Discounterketten) um Marktanteile oder neue Käuferschichten gemeint, zum anderen die Möglichkeit distinktiven ethischen Konsums auf Massenmärkten. Man könnte argumentieren, dass dieser Preis durch die Fair-Trade-Labelling-Organisationen (FLOs) fixiert ist und gerade nicht dem Spiel aus Angebot und Nachfrage unterliegt. Jedoch führt die Besiegelung und die damit einhergehende Entstehung eines Marktes für faire Güter zu einer (wenn auch kontrollierten und organisierten) Differenzierung der Produkte und Preise konkurrierender Anbieter (z. B. Einzelhändler) (Davies 2007). In Abb. 1 ist der Wandel der Feldlogiken der Fair-Handelsbewegung in seiner Verbindung mit den spezifischen Dimensionen der sie repräsentierenden Organisationsformen in idealtypischer Weise dargestellt. Die neue, vermarktlichte Organisationsform unterscheidet sich hinsichtlich der Organisationsziele, der eingesetzten Techniken, der Autoritätsbezie- 
hungen und der Marketing-Strategien von der alten, zivilgesellschaftlichen. Es wird nun eine hohe Marktdurchdringung angestrebt durch verbesserte Produkte und verstärkte Marketinganstrengungen. Die neue Logik wird innerhalb der Organisation durch veränderte Autoritätsbeziehungen sichtbar, die sich in hierarchisch geordneten, spezialisierten und professionalisierten Stellen wie beispielsweise für Marketing, „business development“ oder „,supply chain management“ widerspiegeln. Die durch eine unabhängige Stelle (FLO-cert) durchgeführte Überprüfung und Zertifizierung der Standards kann dabei als technische Neuerung verstanden werden.

Aus einer zivilgesellschaftlichen Logik, die hauptsächlich auf Bürger ausgerichtet ist, wurde eine vermarktlichte Logik, die auf Konsumenten ausgerichtet ist. Der Wandel der Feldlogik transzendiert dabei das engere organisationale Feld der FairHandelsbewegung und erreicht nun auch Marktteilnehmer. Es kann argumentiert werden, dass der Kommodifizierungsprozess nicht nur direkte Auswirkungen auf den Erwerb von fair gehandelten Waren durch Verbraucher hat, sondern auch das Wissen um ethische Qualitäten von Gütern auf Märkten beeinflusst. Institutionelle oder Feldlogiken stellen Handlungsrationalitäten und Skripte bereit, an denen Akteure ihr Handeln orientieren (Meyer 2010). Produktsiegel ermöglichen solch eine Orientierung, da sie klare Signale senden und Informationen auf effiziente, verständliche und konsistente Weise transportieren können. Da Fair-Trade-Güter sogenannte „credence“- oder Glaubensgüter sind, deren moralische bzw. ethische Qualität zumeist nicht aus dem Aussehen oder Gebrauch der Güter selbst ersichtlich wird, sind Produktsiegel eine wichtige Möglichkeit, um diese Qualität zu signalisieren (Feddersen und Gilligan 2001). Eine Voraussetzung für das Funktionieren dieses Signals ist, dass Konsumenten Vertrauen in das Siegel haben. Im Fairen Handel ebenso wie im Bio-Bereich wird daher versucht, dieses Vertrauen durch eine unabhängige, von einer dritten Partei durchgeführte Zertifizierung herzustellen (Koos 2011). Darüber hinaus signalisiert die Besiegelung eine moralische Überlegenheit im Vergleich zu „normalen“ Gütern und hilft somit, Konsumidentitäten auf Basis normativer Ideale zu differenzieren. Das Siegel selbst wird somit zu einem „Framing“-Artefakt. Nicht zuletzt verringern sich durch die breitere Verfügbarkeit von gesiegelten Produkten die Transaktionskosten für den Kauf dieser Produkte.

H1 Die organisierte Vermarktlichung des Fairen Handels durch die Gründung von Fair-Trade-Labelling-Organisationen und die Einführung von Fair-Handels-Siegeln erhöht die Wahrscheinlichkeit, dass Personen über Fairen Handel informiert sind und fair gehandelte Produkte kaufen.

\subsubsection{Mobilisierungsstrukturen und Framing-Strategien}

Nachdem Fair-Trade-Labelling-Organisationen entstanden sind, stellt sich die Frage, wie diese auf die weitere Diffusion von ethischem Konsum einwirken. Die bloße Existenz eines Labels und die veränderte Feldlogik der Fair-Trade-Bewegung reichen möglicherweise nicht aus, um das starke Wachstum des Fairen Handels zu erklären. Warum wächst beispielsweise der Konsum fair gehandelter Produkte in manchen Ländern stärker als in anderen? Die Fair-Trade-Bewegung und vor allem die Fair-Trade-Labelling-Organisationen verwenden zwei Strategien unter Bezug- 
nahme auf zwei zentrale strategische Handlungsfelder der Bewegung, um fairen Handel zu fördern (Eshuis und Harmsen 2003): zum einen das Feld der Verbraucher und die direkte Bezugnahme auf diese durch Kampagnen und Marketing, zum anderen das Feld der Hersteller und Einzelhändler, die fair gehandelte Rohstoffe in ihren Produkten verwenden bzw. gesiegelte Produkte direkt verkaufen. Aus einer sozialen Bewegungsperspektive engagieren sie sich also hauptsächlich auf zwei Arten: durch das Framing für Konsumenten und durch die strategische Erschließung von Mobilisierungsstrukturen (Benford und Snow 2000; McCarthy und Zald 1977).

Das Framing-Konzept der sozialen Bewegungsforschung nimmt an, dass Menschen nur an kollektivem Handeln teilnehmen, wenn ein Phänomen zunächst als „Problem“ verstanden wird und eine Handlungsalternative verfügbar ist, die auf dieses Problem einwirkt. Daher versuchen soziale Bewegungsorganisationen, „Interpretationsschemata“ (Goffman 1975) oder Deutungsmuster der sozialen Welt zu schaffen, die mobilisierend wirken. Die Greenpeace-Kampagne gegen die Versenkung der Ölplattform „Brent Spar“ im Jahre 1995 kann als ein gutes Beispiel zur Illustration dienen. Greenpeace-Aktivisten besetzten die Plattform und riefen zu einem Boykott der Shell-Tankstellen auf. Das Ziel der Kampagne war es, vermittelt durch die Medien ein kollektives Deutungsmuster der Umweltverschmutzung durch Ölkonzerne zu entwickeln. Mit dem Aufruf zu einem Boykott der Shell-Tankstellen verwies Greenpeace aber noch auf eine praktische Möglichkeit, wie dieses Deutungsmuster in eine kollektive Aktion übersetzt werden kann. Der Boykott war ein so großer Erfolg, dass die Ölplattform nicht versenkt wurde.

Im Fall des Fairen Handels versuchen die Fair-Trade-Organisationen (FTOs), die Bedingungen des Welthandels als ein Problem für die weniger entwickelten Länder darzustellen (bzw. zu rahmen). Die FTOs kommunizieren diese Kritik über Flyer, Werbespots, öffentliche Kampagnen oder auch Lehrmaterialien für Schulen. Durch das Angebot an fair gehandelten Produkten bieten sie aber auch Handlungsalternativen an, die eine kollektive Bearbeitung des Problems möglich machen. In modernen Kommunikationsgesellschaften mit immensen Werbemaßnahmen von Konzernen ist es besonders schwierig, alternative Deutungen auf Märkten zu konstituieren und zu vermitteln. Framing-Anstrengungen sind daher besonders wichtig, um die Informationsasymmetrie zwischen Konsument und Verkäufer auszugleichen und die Aufmerksamkeit der Verbraucher auf alternative Möglichkeiten des Konsums zu lenken. Die Art und Weise, wie Fair Trade als Lösung für die Probleme des Welthandels dargestellt oder gerahmt wird, ist sicherlich von entscheidender Bedeutung. Aber auch das Volumen der eigenen Kommunikationsbemühungen als Gegengewicht zu den Werbemaßnahmen der „,normalen Unternehmen“ ist wichtig. Als Hypothese formuliert, lässt sich erwarten:

H2 Wenn Fair-Trade-Organisationen mehr Ressourcen dafür verwenden, über fairen und unfairen Handel zu informieren, sollten mehr Verbraucher über den Fairen Handel informiert sein und verstärkt Fair-Trade-Produkte kaufen.

Eine zweite Perspektive nimmt die Mobilisierungsstrukturen in den Blick. Dabei handelt es sich um ,collective vehicles, informal as well as formal, through which people mobilize and engage in collective action“ (McAdam et al. 1996a, S. 3). Diese 
Strukturen verweisen zum einen auf professionalisierte, mit Ressourcen ausgestattete Bewegungsorganisationen, die Bürger mobilisieren. Zum anderen bezeichnen Mobilisierungsstrukturen informelle Netzwerke, über welche mögliche Teilnehmer angesprochen und in soziale Bewegungen eingebunden werden. Die zugrundeliegende Annahme dabei ist, dass es konkreter Ressourcen (wie z. B. Zeit, Geld oder Computer) bedarf, um Menschen zu koordiniertem kollektivem Handeln zu bewegen. So organisieren Bewegungsorganisationen beispielsweise mithilfe von E-MailVerteilern, sozialen Medien und Plakaten kollektive Aktionen. Darüber hinaus nimmt diese Perspektive auch soziale Beziehungsstrukturen wie Kirchengemeinden oder Nachbarschaftsnetzwerke in den Blick, die als Orte der Mobilisierung genutzt werden. Allgemeiner gesagt, umfassen Mobilisierungsstrukturen also auch ,the range of everyday life micromobilization structural social locations, that are not aimed primarily at movement mobilization“" (McCarthy 1996, S. 141). Im Fall der Fair-HandelsBewegung werden Händler und Orte des Konsums wie Supermärkte, Discounter oder Wochenmärkte zu einem zentralen Bestandteil der Mobilisierungsstrukturen. Die Fair-Trade-Bewegung versucht, die Möglichkeiten zum Kauf von Fair-TradeProdukte zu verbessern, indem die Anzahl an Geschäften und Handelsketten, die diese Produkte anbieten, aber auch die Anzahl an verfügbaren Produkttypen erhöht wird. Die Besiegelung von Produkten erleichtert dies, da fair gehandelte Produkte nun leicht auch auf Massenmärkten vertrieben werden können. Bevor Fair-TradeZertifizierungen eingeführt wurden, war der besondere Charakter dieser Produkte hauptsächlich durch ausgewiesene ethische Orte des Verkaufs wie Weltläden oder durch ethisch integre Verkäufer wie Kirchengruppen verbürgt. Die FTOs zielten somit auf die Verbesserung der „Infrastruktur“ für fairen Konsum, um die Beteiligung am Fairen Handel zu fördern (Southerton et al. 2004). ${ }^{2}$ Dies kann als eine direkte „Lobbying-Strategie“ durch die Fair-Trade-Labelling-Organisationen verstanden werden, die auf Unternehmen ausgerichtet ist, um diese zur Verwendung von FairTrade-Siegeln für ihre Produkte oder zur Aufnahme von Fair-Trade-Produkten in ihr Verkaufssortiment zu bewegen. Haben einige zentrale Akteure diese moralisierten Produkte im Sortiment, müssen ihre Konkurrenten nachziehen. Als beispielsweise der deutsche Discounter Lidl anfing, ein eigenes Fair-Trade-Sortiment anzubieten, nahm mit einiger Zeitverzögerung auch der direkte Konkurrent Aldi fair gehandelte Produkte in sein Sortiment auf. Der aufkommende Wettbewerb auf moralisierten Märkten führt zu einer weiteren Differenzierung und erhöhten Verfügbarkeit von Produkten, sodass ein ethischer Konsum leichter mit „,normalen“ Einkaufs-Routinen vereinbar wird. Als Hypothese formuliert, lässt sich erwarten:

H3 Je mehr Verkaufsstellen für fair gehandelte Produkte existieren, umso mehr Konsumenten wissen um den Fairen Handel und kaufen fair.

\footnotetext{
${ }^{2}$ Dies kann auch als bewusste Schaffung von Opportunitätsstrukturen verstanden werden. Im Gegensatz zur im nachfolgenden Abschnitt vorgestellten Theorie der politischen Opportunitätsstrukturen verstehe ich unter den Mobilitätsstrukturen nicht extern gegebene, sondern aktiv durch die Bewegungsorganisation erschlossene und geschaffene Strukturen, obwohl die Grenze zwischen exogenen und endogenen Strukturen natürlich fließend ist.
} 


\subsection{Opportunitätsstrukturen für Fairen Handel}

Andere externe Opportunitäten, welche die Mobilisierung von Bürgern beeinflussen können, wurden in der Theorie der politischen Opportunitätsstrukturen herausgearbeitet (Meyer 2004). In einer weiten Definition bestimmt Sydney Tarrow (1994, S. 18) politische Opportunitätsstrukturen als ,consistent - but not necessarily formal, permanent or national - dimensions of the political environment which either encourage or discourage people from using collective action". Je nach Forschungsfeld unterscheiden sich die Autoren sehr stark in ihrer Definition politischer Opportunitätsstrukturen, was zu einer gewissen Willkürlichkeit in der Anwendung dieser Theorie geführt hat (Gamson und Meyer 1996). Bemühungen, diesen Ansatz zu präzisieren, führten zu einer Ausdifferenzierung von mindestens zwei anderen Bereichen von Opportunitätsstrukturen (Koos 2012), nämlich kulturellen (McAdam 1996) und ökonomischen Opportunitätsstrukturen (Wahlström und Peterson 2006).

Die politischen Opportunitätsstrukturen selbst können in Bezug auf den FairTrade-Konsum als nachrangig verstanden werden, da Staaten das Fair-Trade-System zumeist nicht direkt fördern oder behindern. Allerdings lassen sich einige Beispiele dafür finden, dass der Faire Handel doch eine staatliche Unterstützung erhielt. So wurde zur Einführung des Max-Havelaar-Siegels in den Niederlanden eine Informationskampagne zum Fairen Handel staatlich subventioniert. Leider kann dieser Aspekt jedoch komparativ nicht hinreichend erfasst und in der quantitativen Analyse berücksichtigt werden.

Man kann argumentieren, dass wirtschaftliche Opportunitätsstrukturen besonders wichtig für die Mobilisierung und den Erfolg einer sozialen Bewegung sind, die Wirtschaftsakteure, Branchen oder Aspekte des wirtschaftlichen Systems adressiert (Koos 2012). Diese Möglichkeiten werden durch die extern gegebenen wirtschaftlichen Ressourcen und Kosten beeinflusst, die mit bestimmten Handlungen einhergehen. Da fair gehandelte Produkte etwas teurer als herkömmlich gehandelte Produkte sind, sollten Menschen aus Ländern mit einem hohen durchschnittlichen Haushaltseinkommen bessere Möglichkeiten haben, sich an dieser Art des ethischen Konsums zu beteiligen.

H4 Je höher das durchschnittliche nationale Haushaltseinkommen ist, umso mehr Menschen sollten fair gehandelte Produkte kaufen.

Die kulturellen Opportunitätsstrukturen werden wiederum durch geteilte Normen, Überzeugungen und Werte bestimmt (Verba 1965). Sie legen fest, welche Art des Verhaltens angemessen ist oder ob kollektives Handeln eher traditionell orientiert ist und durch das bestehende politische System kanalisiert wird. Ronald Inglehart (1997) argumentiert beispielsweise, dass die postmaterialistischen Werte einen zentralen kulturellen Einfluss bei der Etablierung neuer Arten der politischen, aber auch zivilgesellschaftlichen Beteiligung der Bürger hatten. Somit kann erwartet werden, dass Bürger in Ländern mit einer postmaterialistischen zivilgesellschaftlichen Kultur eher neue Arten des Konsums übernehmen als Menschen in Gesellschaften, in denen traditionelle Werte und Normen dominieren. In der empirischen Forschung 
finden sich einige Belege für die Wichtigkeit postmaterialistischer Haltungen für die Erklärung des politischen Konsums (Copeland 2013; Stolle und Hooghe 2004).

H5 Je stärker postmaterialistische Werte in einer Gesellschaft verbreitet sind, umso höher ist die Bereitschaft, Produkte aus Fairem Handel zu erwerben.

\subsection{Individuelle Determinanten des Fair-Trade-Konsums und -Wissens}

Neben den Opportunitäten und Mobilisierungsmechanismen sollen auch individuelle Charakteristika berücksichtigt werden, die ethischen Konsum erklären können. ${ }^{3}$ So sind Mobilisierungsstrukturen und Framing-Aktivitäten typischerweise ja auf bestimmte Personengruppen ausgerichtet. Da der Fokus dieses Beitrages jedoch auf dem Einfluss des Vermarktlichungsprozesses liegt, werden individuelle Determinanten des Fair-Trade-Konsums nur sehr knapp diskutiert. Individuelle Ansätze zur Erklärung des Konsums von fairen Produkten oder des ethischen Konsums im Allgemeinen fokussieren auf zwei Dimensionen, die Handeln konstituieren und zentral für alle Handlungstheorien sind (Rössel 2008): Auf der einen Seite wird angenommen, dass der Raum an Handlungsmöglichkeiten durch bestimmte Ressourcen oder Restriktionen begrenzt wird (Elster 1989; Verba et al. 1995). Auf der anderen Seite wird angenommen, dass Handeln durch bestimmte Motivationen, Identitäten oder Überzeugungen orientiert und geleitet wird (Opp 1999). Diese Faktoren sind wiederum eng verknüpft mit den sozialen Positionen der Akteure innerhalb eines sozialen Raums (Bourdieu 1984).

Die Ressourcen-Dimension verweist auf materielle Faktoren wie Haushaltseinkommen oder immaterielle Aspekte wie Bildung (Verba et al. 1995). Es wird angenommen, dass eine Knappheit an Ressourcen individuelle Wahlmöglichkeiten des Konsums und der politischen Partizipation beschränkt. Da fair gehandelte Produkte teurer als „,normale“ Güter sind, erleichtert ein höheres Einkommen die Unterstützung des Fairen Handels. Bildung (verstanden als Ressource oder Fähigkeiten) spielt eine zentrale Rolle dabei, Menschen zu helfen, die Komplexitäten des modernen Markttausches und seiner Folgen zu verstehen. ${ }^{4}$ Vorhandene Studien zeigen, dass Personen mit höheren Bildungsabschlüssen mit höherer Wahrscheinlichkeit politisch oder nachhaltig konsumieren (Copeland 2013; Stolle und Hooghe 2004).

Die motivationale Dimension spielt eine entscheidende Rolle bei der Ausbildung und Realisierung einer Handlungsabsicht (Hechter 1994). Selbst einem habitualisierten Handeln liegt eine bestimmte erlernte Handlungsmotivation oder eine zu einem früheren Zeitpunkt durchdachte Handlungsabsicht zugrunde, welche im Handeln nicht bewusst reflektiert werden muss. Empirische Untersuchungen haben die Bedeutung von postmaterialistischen Werthaltungen (Stolle et al. 2005) und genera-

\footnotetext{
3 Die Determinanten des Wissens um Fairen Handel werden hier nicht explizit diskutiert. Ich gehe davon aus, dass die angeführten Charakteristika ethischer Konsumenten zumeist auch mit einem gesteigerten Wissen über Fair Trade einhergehen.

4 Die Abgrenzung zu bestimmten Motivationen oder Überzeugungen, die mit einem bestimmten Bildungsgrad einhergehen, ist hier sicherlich nicht trennscharf; der Einfluss der Bildung könnte auch dem Bereich der Motivationen zugeordnet werden.
} 
lisiertem Vertrauen für die Teilnahme an politischem Konsum gezeigt (Neilson und Paxton 2010). Auch die politische Identität und Orientierung von Personen könnte deren Entscheidung für einen politischen Konsum beeinflussen. Personen, die sich auf einer Rechts-links-Skala politischer Orientierungen als links einordnen und damit zentralen Annahmen kapitalistischer Ökonomien kritischer gegenüberstehen, sind eher bereit, fair gehandelte Produkte zu erwerben.

Die vorliegenden Forschungsergebnisse zeigen zudem, dass die soziale Klassenzugehörigkeit eine wichtige Rolle sowohl für die politische Partizipation als auch für Konsumentscheidungen spielt. Statusunterschiede werden entlang von sozialen Klassenlagen auch durch Konsummuster signalisiert und reproduziert (Bourdieu 1984). Diese Distinktionsbemühungen der Konsumenten zeigen sich auch bei der Entscheidung für oder gegen einen ethischen Konsum (Koos 2012). Personen aus höheren Klassen setzen sich durch den Kauf von ethischen Produkten bewusst von anderen Klassen ab. Wenn, wie oben angedeutet, die Vermarktlichung die Möglichkeit distinktiven Konsums erhöht, sollte sich dies auch in den Effekten der Klassenvariablen widerspiegeln. In Ländern, in denen der organisierte Vermarktlichungsprozess von Fair-Trade-Produkten stattgefunden hat, sollte der Effekt der sozialen Klasse stärker sein als in Ländern, die nicht über ein Fair-Trade-Label verfügen (Crosslevel-Interaktion).

\section{Daten, Variablen und Methode}

\subsection{Daten}

Für die nachfolgende europäisch vergleichende Mehrebenen-Analyse wurden Daten aus der Eurobarometerserie der Europäischen Kommission verwendet. Die Eurobarometer-Umfrage 47.0 von 1997 (Melich 1997) in 15 Ländern ist die bislang einzige existierende Individualdatengrundlage, um den Konsum fairer Produkte aus einer europäisch vergleichenden Perspektive untersuchen zu können. Die Umfrage ist sehr gut für das Erkenntnisinteresse dieser Studie geeignet, da die Befragung zu einem Zeitpunkt durchgeführt wurde, an dem es zwar in allen betrachteten Ländern eine Fair-Trade-Bewegung und fair gehandelte Güter, aber nicht überall FairTrade-Labelling-Organisationen gab. Auf diese Weise ist die Variation für das zentrale Explanans dieser Studie - die organisierte Vermarktlichung - gewährleistet. Aufgrund von Datenbeschränkungen (fehlende Daten) wurden Portugal und Griechenland nicht in der Analyse berücksichtigt. Eine deskriptive grafische Analyse zeigt, dass die Niederlande ein starker Ausreißer sind und die Regressionsergebnisse verzerren (siehe auch Linton et al. 2004). Die Niederlande weichen aufgrund ihrer Vorreiterrolle auf allen erfassten Variablen stark von den restlichen Fällen ab. Aus diesem Grund wurden die Niederlande aus der Analyse ausgeschlossen, um eine 
Verzerrung zu vermeiden. ${ }^{5}$ Nach Rekodierung und Säuberung der Daten verbleiben 12.140 Befragte aus 12 Ländern für die Analyse. Die Länder sind Österreich, Belgien, Dänemark, Finnland, Frankreich, Deutschland, Großbritannien, Irland, Italien, Luxemburg, Schweden und Spanien.

Der Individualdatensatz wurde anschließend mit Daten zur Organisation der FairTrade-Bewegung sowie anderen Makrodaten zu den Opportunitätsstrukturen verknüpft. Die Organisationsdaten wurden aus einem Bericht über den Fair-Trade-Sektor in Europa gewonnen (Martinelli 1998). Dieser Bericht basiert auf einer Umfrage unter Fair-Trade-Organisationen in 16 Ländern aus dem Jahr 1997 und beinhaltet zentrale Informationen über die nationale Struktur der Fair-Trade-Bewegung wie zentrale Organisationen, Anzahl an Weltläden, Ausgaben für Kampagnen und Marketing sowie kurze historische Abrisse über die Bewegung in jedem Land. Fehlende Informationen für einige Variablen wurden aus einem früheren Bericht von 1995 hinzugefügt (Martinelli 1995). Weitere Daten wie z. B. zum Gründungsjahr der Label und der Bewegung insgesamt wurden aus späteren Berichten gewonnen (Krier 2001, 2006, 2007). Durch einen Vergleich der Berichte über die Zeit und zusätzliche Recherchen nationaler Quellen (wie Webseiten der Label-Organisationen und Importorganisationen) wurden die Daten so weit als möglich validiert.

\subsection{Operationalisierungen}

\subsubsection{Variablen auf der Individualebene}

Das Ziel der Fair-Trade-Bewegung ist es, Menschen über die Probleme des Welthandels aufzuklären sowie den Absatz fair gehandelter Produkte zu erhöhen. Aus diesem Grund werden zwei abhängige Variablen in der Analyse verwendet, die das Wissen (FH Wissen) um und den Kauf von Fair-Handels-Produkten (FH Konsum) abbilden. Beide Variablen basieren auf folgender Frage in der Eurobarometer-Umfrage (Melich 1997, S. 419): „Einige Produkte aus Entwicklungsländern tragen ein ,Fairer Handel '-Zeichen. Dieses Zeichen garantiert, dass die Produkte unter Gewährleistung fairer Arbeitsbedingungen für Landwirte und Arbeitnehmer und die Umwelt hergestellt wurden. Es bedeutet auch, dass ein fairer Teil des Gewinns zurück zu dem Produzenten bzw. den Arbeitnehmern fließt. Haben Sie schon einmal von dieser Art von Produkten gehört oder nicht? Und haben Sie diese Art von Produkten jemals gekauft?" Es wurden zwei Dummy-Variablen erstellt ( $1=$ ja; $0=$ nein $)$, welche zum einen den Kauf und zum anderen das Wissen über Fair Trade abbilden. Ein Nachteil dieser Frage ist, dass die Häufigkeit des Kaufes nicht erfasst wurde.

Die oben beschriebene Ressourcen-Dimension wurde operationalisiert über das Haushaltseinkommen und das Bildungsniveau. Das Einkommen wird anhand des relativen Haushaltsäquivalenzeinkommens (neue OECD-Skala) (Hagenaars et al. 1994) erfasst. Da sich die Einkommensskalen in der Eurobarometer-Umfrage zwi-

\footnotetext{
${ }^{5}$ Eine Schätzung ohne die Niederlande erlaubt eine konservativere und robustere Einschätzung der zugrundeliegenden Triebkräfte des Fairen Handels. Wenn man die Niederlande in die Analyse mit einbezieht und durch eine Variable auf den spezifisch niederländischen Effekt kontrolliert, bleiben die Analysen jedoch konsistent.
} 
schen den Ländern leicht unterscheiden, wird der Mittelpunkt jeder Einkommenskategorie verwendet, um das Haushaltsäquivalenzeinkommen im Vergleich zum nationalen Durchschnittseinkommen für jeden Befragten zu berechnen. Um ein vergleichbares $\mathrm{Ma} \beta$ zu erhalten, wurden die resultierenden Werte in vier Einkommensgruppen unterteilt, bestehend aus Menschen, die weniger als $80 \%$, 80 bis $120 \%$ und über $120 \%$ des durchschnittlichen nationalen Haushaltseinkommens verdienen, zuzüglich einer Kategorie für fehlende Werte (missings). Bildung wird durch eine Gruppe von drei Dummy-Variablen operationalisiert, die primäre, sekundäre und tertiäre Bildung unterscheiden. Die Bildungsvariable basiert auf einer Eurobarometer-Frage, die das Alter, zu dem die Ausbildung abgeschlossen war, erfasst (leider ist dies die einzige Möglichkeit, den Bildungsgrad in Eurobarometer-Umfragen zu operationalisieren). Beendeten die Befragten ihre Schulbildung vor dem 15. Lebensjahr, wird dies als primäre Bildung kodiert, ein Alter von 15 bis 19 Jahren wird als sekundäre Bildung erfasst, und Personen, die nach dem 19. Lebensjahr ihre Ausbildung abgeschlossen haben, werden in die tertiäre Bildungskategorie eingruppiert (siehe auch Pfeiffer 2009).

Da in der Eurobarometer-Umfrage leider keine Wertorientierungen abgefragt wurden, werden hier zwei Variablen verwendet, die das generalisierte transnationale Vertrauen sowie die politische Selbsteinschätzung auf einer Rechts-links-Skala abbilden. Die Variable generalisiertes transnationales Vertrauen misst das Vertrauen der Befragten in Menschen aus dem eigenen Land und anderen Ländern. Das Maß basiert auf folgender Frage: „Jetzt möchte ich Sie fragen, wie viel Vertrauen Sie in Menschen aus verschiedenen Ländern haben. Sagen Sie mir bitte für jedes Land, ob Sie dazu neigen, diesen Menschen zu vertrauen oder eher nicht?" Die angezeigte Liste bestand aus Personen aus 18 europäischen Ländern, ergänzt durch Russland und die USA, einschließlich des Landes des Befragten. Um einen Index zu bilden, der dem Problem der Antwortverweigerung für einzelne der abgefragten Länder gerecht wird, wurde die Anzahl an positiven Antworten (Vertrauen in andere) durch die Anzahl an allen gegebenen Antworten (sowohl Vertrauen als auch kein Vertrauen in andere, ohne die Kategorie „weiß nicht“) geteilt. Die Variable bildet also das relative standardisierte Vertrauen gegenüber Menschen aus dem eigenen Land und anderen Ländern ab. Als zweites motivationales Maß wurde die politische Orientierung auf einer Rechts-links-Skala verwendet. Die Variable basiert auf folgender Frage: „In politischen Angelegenheiten sprechen Menschen oft von ,links' und ,rechts'. Wie würden Sie Ihre Auffassung auf dieser Skala einordnen?" Die Befragten wurden gebeten, sich auf einer Zehn-Punkte-Skala einzuordnen, die auf einer Karte dargestellt wurde, wobei 1 ,links“ entsprach und 10 „rechts“. Die Antworten wurden in drei Dummy-Variablen rekodiert, für ,links“ (Skalenwerte von 1 bis 4), „mitte“ (Werte 5 bis 6) und ,rechts“" (Werte 7 bis 10), sowie eine Dummy-Variable für die zahlreichen fehlenden Werte bei dieser Frage.

Die Variablen für soziale Klasse basieren auf dem „European Socio-economic Classification (ESeC)"-Schema (Rose und Harrison 2007). Die ESeC-Klassifikation folgt grundsätzlich dem von Robert Erikson und John H. Goldthorpe (1992) entwickelten Klassenschema, das Personen hinsichtlich ihrer beruflichen Stellung kategorisiert. Ich unterscheide die Dienstklasse, höhere Angestellte, Selbstständi- 
ge (einschließlich Landwirte), Facharbeiter (als eine Kategorie) und Arbeiter ohne Ausbildung (Rose und Harrison 2007, S. 464).

Darüber hinaus wird für Geschlecht und Alter kontrolliert. Es gibt zahlreiche Studien, die zeigen, dass Frauen häufiger politisch oder ethisch einkaufen als Männer (Koos 2012; Micheletti 2004; Stolle et al. 2005). Im Hinblick auf den Effekt des Lebensalters kaufen sehr junge und sehr alte Menschen eher weniger politisch orientiert als Menschen im mittleren Alter (von 26 bis 55) (Strømsnes 2005). Daher wurde die Variable Alter in Jahren zusätzlich als quadrierter Term in die Regression eingeführt, um den potenziell kurvilinearen Effekt von Alter abzubilden.

\subsubsection{Kontextuelle Variablen}

Auf der kontextuellen (Länder-)Ebene wurden mehrere Variablen operationalisiert, die organisatorische Determinanten und Gelegenheitsstrukturen des Fair-Trade-Konsums messen sollen. Erstens gibt die Variable organisierte Vermarktlichung (Dummy-Variable) an, ob eine Fair-Trade-Labelling-Organisation in einem Land vorhanden ist und ob diese Organisation erfolgreich aktiv ist, d.h. gelabelte Produkte auch verfügbar sind $(1=\mathrm{ja} ; 0=$ nein $)$. Die Mehrheit der 12 Länder verfügte über eine Fair-Trade-Labelling-Organisation, mit Ausnahme von Finnland und Spanien sowie Frankreich, wo eine FLO zwar 1992 gegründet wurde, diese aber erst 1998 mit der Besiegelung begann. Zweitens wird die organisierte Verfügbarkeit von Fair-TradeProdukte gemessen als Anzahl an Verkaufspunkten (insbesondere Supermärkte - ohne Weltläden) (Martinelli 1998), standardisiert anhand der Bevölkerungsgröße, um die Daten länderübergreifend vergleichbar zu machen. Drittens werden die FramingAnstrengungen der Fair-Trade-Bewegungen operationalisiert durch das Budget für Kampagnen, Öffentlichkeitsarbeit und Marketing (Martinelli 1995, 1998), wiederum standardisiert anhand der Bevölkerungsgröße. Abschließend wird aufgrund möglicher Lebenszyklus-Effekte der differentiellen Gründung und Verbreitung der FairTrade-Bewegung auch das Alter der Fair-Trade-Bewegung (in Jahren) kontrolliert. Das Gründungsjahr der Fair-Trade-Bewegung in jedem Land wurde durch den Zeitpunkt der Errichtung der ersten Fair-Trade-Organisation (Import-Organisation oder Welt-Shop) bestimmt.

Die Opportunitätsstruktur für ethischen Konsum wurde durch den Reichtum und die post-materialistische Kultur eines Landes erfasst. Der Wohlstand eines Landes wurde operationalisiert durch den Anteil der Haushaltsausgaben für Lebensmittel im Vergleich zu allen anderen Ausgaben, z. B. für Wohnen, Gesundheit usw. Dies wurde mit den durch Eurostat (2009) bereitgestellten Daten des Household Budget Survey von 1994 erfasst. Der Anteil wurde von 100 abgezogen, sodass ein höherer Wert einen höheren Wohlstand anzeigt. Diese Variable ist ein spezifischeres Maß für die Freiheitsgrade bei Konsumentscheidungen als das Bruttoinlandsprodukt (BIP) pro Kopf (Martín-Lagos López 2010). Jedoch wurden alle Analysen auch mit dem BIP pro Kopf des Jahres 1997 geschätzt (nicht berichtet), und die Ergebnisse sind konsistent. Die post-materialistische Kultur eines Landes wird gemessen über den Anteil der Menschen innerhalb eines Landes, die sich als Postmaterialisten identifizieren. Diese Variable basiert auf einer anderen Eurobarometer-Umfrage von 1997 (47.1), in welcher Ingleharts Standard-Postmaterialismus-Items verwendet wurden. 
Die kontextuellen Variablen wurden mit den Individualdaten verknüpft, sodass eine gemeinsame Analyse möglich ist.

Abschließend wird noch eine explorative vergleichende Analyse von Deutschland und Großbritannien über die Zeit für die 2000er-Jahre durchgeführt, um die Wachstumsdynamik des Fairen Handels zu analysieren. Dazu wurden Daten aus den oben genannten Berichten über Fair Trade in Europa herangezogen (Krier 2001, 2006, 2007). Diese wurden um Informationen aus den Jahresberichten der FLO (FLO 2006, 2008), ein Maß für das BIP pro Kopf in Kaufkraftparitäten (BIP pro Kopf in KKP) sowie den Anteil an Postmaterialisten (aus verschiedenen EurobarometerUmfragen) ergänzt.

\subsection{Methode}

Um die Mehrebenen-Struktur der Daten zu berücksichtigen, wurden logistische hierarchische Regressionsmodelle geschätzt (Guo und Zhao 2000). Da die Individuen in Ländern eingebettet sind und anzunehmen ist, dass sich die Individuen innerhalb von Ländern stärker ähneln als zwischen den Ländern, ist die Annahme der Unabhängigkeit der Beobachtungen verletzt, was zu verzerrten Standardfehlern und damit auch zu verzerrten Signifikanztests führt. Mehrebenen-Modelle adressieren dieses Problem und erlauben es, den Einfluss von Individualmerkmalen und von Kontextmerkmalen gleichzeitig zu schätzen (Hox 2002). Eine Korrelationsanalyse der Makrovariablen ergab, dass die verwendeten Maße nicht stark miteinander zusammenhängen (Pearsons $r<0,6$ ). Für die vergleichende Analyse der weiteren Entwicklung des Fairen Handels wurden die zwei Länder Deutschland und Großbritannien ausgewählt. In beiden Ländern wurde das Fair-Trade-Siegel zu einem ähnlichen Zeitpunkt begründet (Deutschland 1992; Großbritannien 1994). Die Entwicklung des Fairen Handels verlief aber seit Anfang 2000 unterschiedlich, sodass hier explorativ die Wirkung verschiedener Determinanten untersucht werden kann.

\section{Wissen um Fairen Handel und Fair-Trade-Konsum im europäischen Vergleich}

In Abb. 2 werden die Anteile der Befragten je Land ausgewiesen, die zum Befragungszeitpunkt 1997 Fair-Trade-Produkte gekauft haben oder über das Fair-Handelssystem informiert sind. Es lässt sich erkennen, dass in den Niederlanden sowohl der Fair-Trade-Konsum als auch die Kenntnisse über den Fairen Handel am weitesten verbreitet sind. Da sich die Niederlande auch bei den unabhängigen Variablen deutlich von den anderen Ländern unterscheiden, wird das Land aus der weiteren Analyse ausgeschlossen. Für die übrigen Länder lässt sich eine große Variation im Fair-Trade-Wissen und -Konsum ersehen. In Luxemburg kennt mehr als die Hälfte der Bevölkerung den Fairen Handel, und fast ein Drittel der Bevölkerung hat tatsächlich Fair-Trade-Produkte gekauft. Mit $15 \%$ hat Großbritannien nur die Hälfte des Anteils der Fair-Trade-Konsumenten von Luxemburg, jedoch kennen $40 \%$ der Befragten den Fairen Handel. Etwas geringer sind das Wissen und der Konsum von fair gehandelten Produkten in den mitteleuropäischen Ländern wie Deutschland 


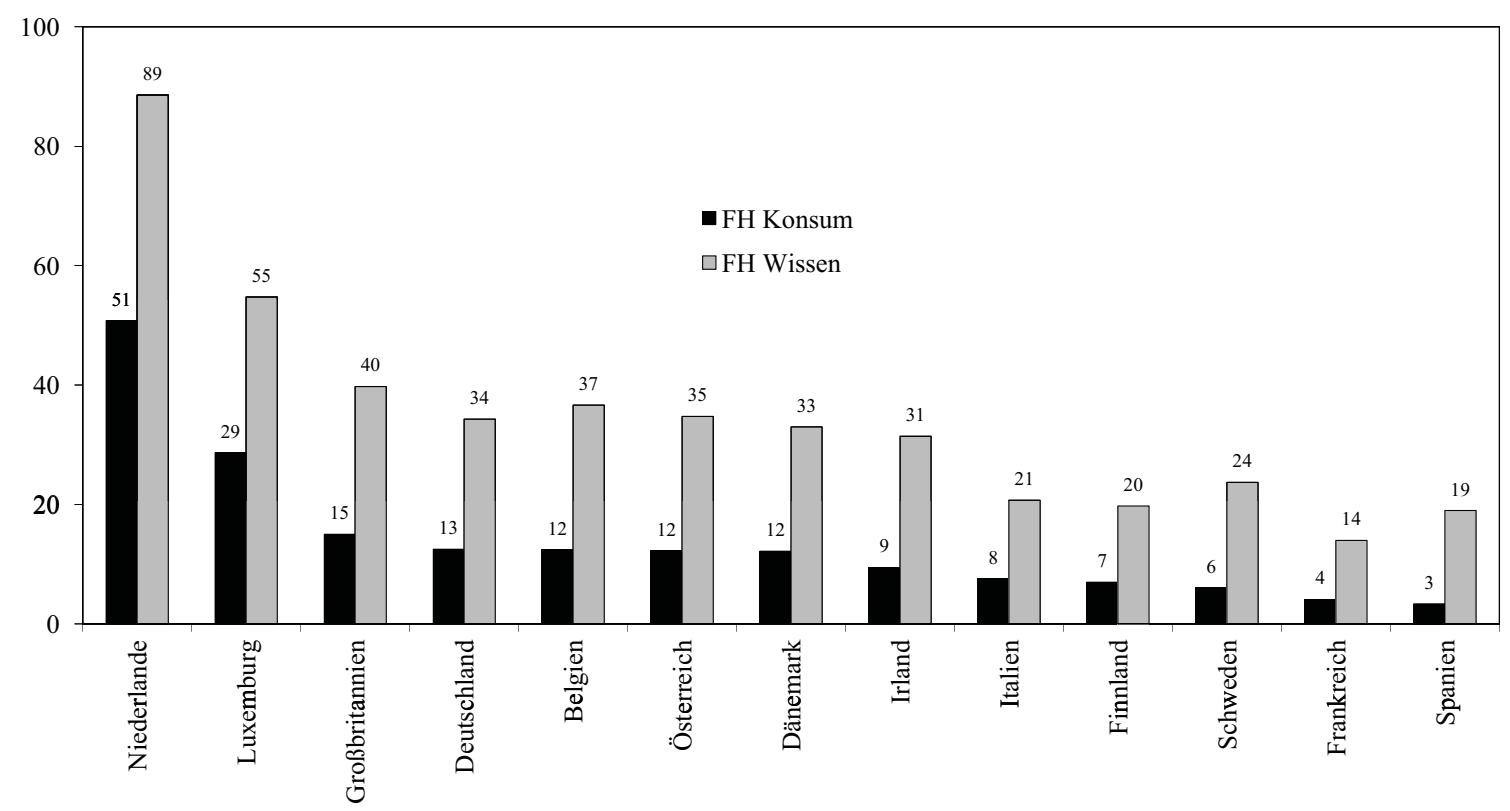

Abb. 2 Anteil der Befragten, die Fair-Trade-Produkte gekauft haben und die die Bedeutung des Fairen Handels kennen (in \%) (Quelle: Eurobarometer 47.0 (Melich 1997); eigene Berechnungen)

und Belgien, aber auch in Irland und Dänemark. Am geringsten sind sie in den nördlichen und südlichen europäischen Ländern sowie in Frankreich. Dort kennen nur $14 \%$ der Befragten den Fairen Handel und $4 \%$ kaufen Fair-Trade Produkte. In Spanien kaufen nur $3 \%$ der Befragten Fair-Trade-Produkte, aber fast ein Fünftel der Bevölkerung kennt den Fairen Handel. Die niedrigen Werte bei den skandinavischen Ländern scheinen etwas überraschend, da diese in anderen Studien sehr hohe Werte des politischen Konsums aufweisen (Koos 2012). Jedoch lässt sich hier ein erster Hinweis auf mögliche Auswirkungen organisatorischer Unterschiede in den FairTrade-Bewegungen zum damaligen Zeitpunkt finden. In den Ländern ohne eine eigene Fair-Trade-Labelling-Organisation finden sich weniger Personen, die über den Fairen Handel informiert sind und solche Produkte kaufen. Um die Hypothesen zu überprüfen und die Unterschiede besser erklären zu können, werden nachfolgend die multivariaten Ergebnisse vorgestellt.

Tab. 1 zeigt die Ergebnisse der logistischen Mehrebenen-Regressionen für den Fair-Trade-Konsum (Modell 1) und das Wissen über Fairen Handel (Modell 2). Hinsichtlich des Geschlechterunterschiedes finden wir, dass Frauen statistisch signifikant häufiger Fair-Trade-Produkte kaufen als Männer, jedoch findet sich dieser Unterschied nicht für die Wissensdimension. Die Ergebnisse der Altersvariablen bestätigen den erwarteten umgekehrt u-förmigen Zusammenhang für beide abhängigen Variablen. Zunächst nimmt sowohl die Wahrscheinlichkeit des Wissens als auch des Konsums mit steigendem Alter zu. Für Personen, die älter sind als 45 Jahre, nimmt sie für beides dann wieder ab. Wie erwartet, finden wir einen positiven Bildungseffekt. Befragte mit einem höheren Bildungsabschluss (oder mehr Bildungsjahren) weisen eine höhere Wahrscheinlichkeit auf, über Fair-Trade-Produkte Bescheid zu wissen oder diese zu kaufen, als Befragte, die über einen primären Bildungsabschluss verfügen (Referenzkategorie). Befragte mit einem höheren Einkommen (mehr als $120 \%$ des nationalen Durchschnittseinkommens) haben eine höhere Chance, Fair- 
Tab. 1 Logistische Mehrebenenregression Fair-Trade-Konsum und Wissen über Fairen Handel (MikroEbene)

\begin{tabular}{|c|c|c|c|c|}
\hline \multirow{3}{*}{ Konstante } & \multirow{2}{*}{\multicolumn{2}{|c|}{$\begin{array}{l}\text { Modell } 1 \\
\text { FH Konsum }\end{array}$}} & \multirow{2}{*}{\multicolumn{2}{|c|}{$\begin{array}{l}\text { Modell } 2 \\
\text { FH Wissen }\end{array}$}} \\
\hline & & & & \\
\hline & $-4,60 * * *$ & $(0,38)$ & $-2,35 * * *$ & $(0,26)$ \\
\hline Weiblich & $0,23 * * *$ & $(0,07)$ & 0,03 & $(0,04)$ \\
\hline Alter & $0,05^{* * *}$ & $(0,01)$ & $0,04 * * *$ & $(0,01)$ \\
\hline Alter $^{2}$ & $-0,00 * * *$ & $(0,00)$ & $-0,00 * * *$ & $(0,00)$ \\
\hline \multicolumn{5}{|c|}{ Bildungsniveau (Ref.: primär) } \\
\hline Sekundäre Bildung & $0,21^{*}$ & $(0,10)$ & $0,15^{*}$ & $(0,06)$ \\
\hline Tertiäre Bildung & $0,72 * * *$ & $(0,11)$ & $0,54 * * *$ & $(0,07)$ \\
\hline \multicolumn{5}{|c|}{ Relatives Haushaltseinkommen (Ref.: $<80 \%$ ) } \\
\hline $80-120 \%$ & 0,10 & $(0,09)$ & $0,10^{+}$ & $(0,06)$ \\
\hline Mehr als $120 \%$ & $0,20 *$ & $(0,09)$ & $0,17 * *$ & $(0,06)$ \\
\hline Missing & $-0,11$ & $(0,09)$ & $0,10^{+}$ & $(0,06)$ \\
\hline \multicolumn{5}{|c|}{ Soziale Klasse (Ref.: unqualifizierte Arbeiter) } \\
\hline Service-Klasse & $0,81 * * *$ & $(0,11)$ & $0,57 * * *$ & $(0,07)$ \\
\hline Höhere Angestellte & $0,47 * * *$ & $(0,11)$ & $0,39 * * *$ & $(0,07)$ \\
\hline Selbstständige \& Bauern & 0,25 & $(0,15)$ & $0,25 * *$ & $(0,09)$ \\
\hline Niedrige Angestellte & $0,42 * *$ & $(0,13)$ & $0,29 * * *$ & $(0,08)$ \\
\hline Studierende & $0,69 * * *$ & $(0,15)$ & $0,44 * * *$ & $(0,10)$ \\
\hline Arbeitslose & 0,06 & $(0,15)$ & 0,04 & $(0,09)$ \\
\hline Hausperson & 0,18 & $(0,13)$ & 0,08 & $(0,08)$ \\
\hline \multicolumn{5}{|c|}{ Politische Orientierung (Ref.: rechts) } \\
\hline Links & $0,36 * * *$ & $(0,09)$ & $0,15^{*}$ & $(0,06)$ \\
\hline Mitte & 0,01 & $(0,09)$ & 0,01 & $(0,06)$ \\
\hline Missing & $-0,08$ & $(0,11)$ & $-0,16^{*}$ & $(0,07)$ \\
\hline Generalisiertes Vertrauen & $0,40 * *$ & $(0,12)$ & $0,26 * *$ & $(0,08)$ \\
\hline Log Likelihood & $-3768,84$ & & $-6962,71$ & \\
\hline BIC & 7735,18 & & $14.122,91$ & \\
\hline
\end{tabular}

Standardfehler in Klammern, Signifikanzniveaus ${ }^{+} p<0,10, * p<0,05, * * p<0,01, * * * p<0,001$ Quelle: Eurobarometer 47.0 (Melich 1997), eigene Berechnungen

Trade-Güter zu kaufen, als Personen mit einem niedrigeren Einkommen (weniger als $80 \%$ ). Dies zeigt sich interessanterweise auch für die Wissensdimension.

Hinsichtlich der motivationalen Determinanten zeigt sich, dass Befragte, die sich politisch eher links einordnen, eine höhere Wahrscheinlichkeit aufweisen, über Fairen Handel informiert zu sein und solche Produkte zu kaufen. Dies ist kaum verwunderlich, da der Faire Handel eine Kritik der liberalen Marktwirtschaft impliziert. Schließlich sind die Befragten, die mehr Vertrauen gegenüber Menschen aus anderen Ländern haben, eher bereit, fair gehandelte Produkte zu kaufen, und sie sind häufiger über Fairen Handel informiert.

Auch die soziale Klassenposition zeigt für beide abhängigen Variablen einen statistisch signifikanten Einfluss. Mitglieder der Dienstklasse und höhere Angestellte haben im Vergleich zu nicht-qualifizierten Arbeitern die höchste Chance, Fair-TradeProdukte zu kennen und diese auch zu kaufen. Während kein signifikanter Effekt für 
die Gruppen der Selbstständigen (nur für Kauf), der Hauspersonen und Arbeitslosen $\mathrm{zu}$ beobachten ist, weisen vor allem Studierende eine höhere Wahrscheinlichkeit auf, über Fairen Handel informiert zu sein und diese Produkte zu kaufen. Insgesamt bestätigen diese Befunde zu den individuellen Determinanten neuere Studien zum Konsum fair gehandelter Produkte (Arndorfer und Liebe 2013; Sunderer und Rössel 2012).

Im Mittelpunkt dieser Analyse stehen jedoch die kontextuellen Einflussfaktoren auf das Wissen und den Kauf fair gehandelter Produkte, die im Folgenden näher betrachtet werden. Da sich keine signifikanten Veränderungen der Individualeffekte finden, werden diese für die kommenden Modelle zwar mitgeschätzt, jedoch nicht berichtet. In den Modellen 3 und 4 (vgl. Tab. 2) wird die Hypothese H1 überprüft, dass die organisierte Vermarktlichung des Fairen Handels die Wahrscheinlichkeit erhöht, Fair-Trade-Produkte zu kaufen und über diese informiert zu sein. Es findet sich ein statistisch signifikanter positiver Effekt der organisierten Vermarktlichung auf beide abhängigen Variablen. Die Gründung einer Fair-Trade-Labelling-Organisation und die damit verbundene Einführung von Produktsiegeln für fair gehandelte Produkte erhöht also die Wahrscheinlichkeit, dass Konsumenten in einem Land über diese Produkte informiert sind und diese auch erwerben. In Hypothese H2 wurde erwartet, dass die spezifischen nationalen Mobilisierungsstrukturen - im Sinne der Verfügbarkeit der Produkte (operationalisiert als Anzahl an Verkaufsstellen pro Kopf) - einen Einfluss auf den Fair-Handels-Konsum und das Wissen haben. Diese Variable hat jedoch keinen statistisch signifikanten Einfluss. Somit wirkt sich also vor allem die Gründung der Fair-Trade-Labelling-Organisationen, der damit einhergehende Wandel der Feldlogik sowie die Bereitstellung von Produktsiegeln auf die beiden betrachteten Dimensionen der Etablierung des Fairen Handels aus. Durch die Aufnahme der Fair-Trade-Produkte in den Massenmarkt und das leicht verständliche Siegel erhöht die Vermarktlichung sowohl das Wissen über Fair Trade als auch den Konsum fair gehandelter Produkte.

Es lässt sich argumentieren, dass die Einführung von Fair-Trade-Siegeln die Voraussetzung für eine größere Verfügbarkeit und Bekanntheit dieser Produkte geschaffen hat. Durch die Siegel lassen sich die Produkte auch unabhängig vom Verkaufsort (z. B. einem Weltladen) als fair gehandelt erkennen. Wie die empirischen Ergebnisse zeigen, reicht jedoch die durch die Besiegelung erhöhte Verfügbarkeit der fair gehandelten Produkte im Jahr 1997 allein nicht aus, um die Länderunterscheide hinsichtlich des Wissens um Fairen Handel und des Kaufs fair gehandelter Produkte erklären zu können. Zu diesem frühen Zeitpunkt der Bewegung scheint die Verschiebung der Feldlogik von einer ideell motivierten zivilgesellschaftlichen hin zu einer Marktlogik einen stärkeren Einfluss auf den Kauf und das Wissen zu haben. Dies lässt sich am Beispiel der Gründung des niederländischen Fair-Trade-Siegels „,Max Havelaar" illustrieren. So berichten die Fair-Trade-Aktivisten Fenny Eshuis und Jos Harmsen (2003), dass die Gründung der Max-Havelaar-Organisation und die damit verbundene Einführung des Siegels eine enorm große Aufmerksamkeit bei den Bürgern, in den Medien und bei Unternehmen hervorrief. Die neue Marktgängigkeit des Fairen Handels führte zu einem sprunghaften Anstieg des Marktanteils des ersten gesiegelten Fair-Trade-Produktes - einem Kaffee -, ohne dass die Verfügbarkeit dieses Produktes in den Geschäften zu Anfang stark gestiegen war. 


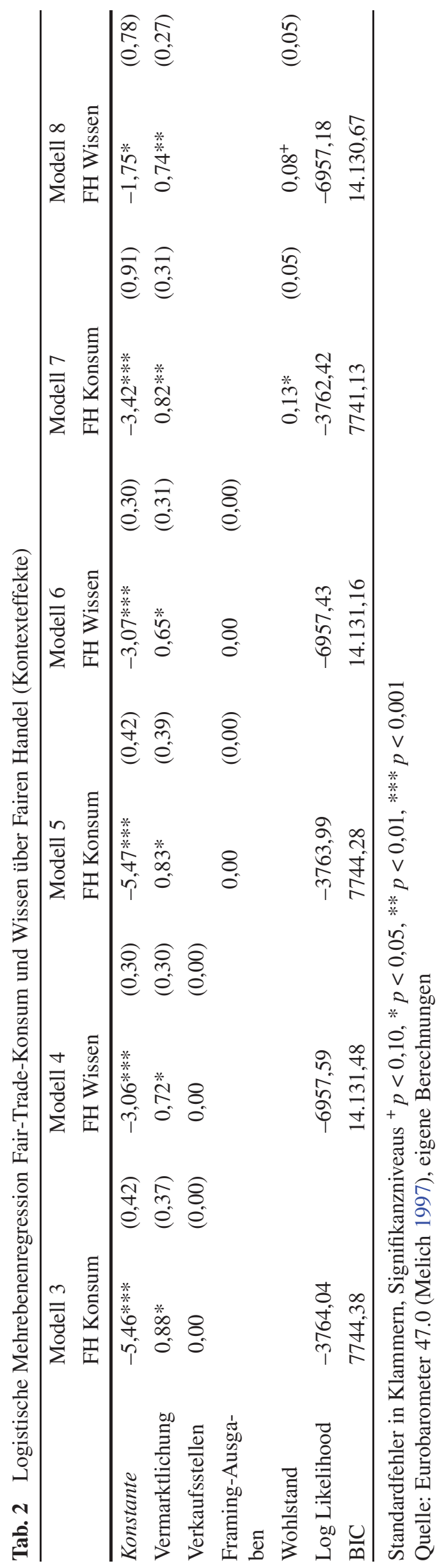


In den Modellen 5 und 6 wird die Hypothese überprüft, dass das Ausmaß der Framing-Bemühungen einen positiven Einfluss auf die Entstehung des ethischen Konsums hat. Es zeigt sich, dass die Ausgaben für Kampagnen keinen statistisch signifikanten Effekt auf den Kauf von Fair-Trade-Produkten und das Wissen über sie haben. Hier muss jedoch berücksichtigt werden, dass nicht der Einfluss spezifischer Interpretationsschemata, sondern nur das Ausmaß der Framing-Bemühungen untersucht wurde. In alternativen Modellen wurde auf Basis einer Zeitungsanalyse die nationale Berichterstattung über Fairen Handel und somit die mediale Resonanz als Framing-Indikator verwendet. Auch diese Analyse erbrachte keinen statistisch signifikanten Einfluss des Framings auf den Konsum oder das Wissen. ${ }^{6}$ Als nächstes wurden die Hypothesen für den Einfluss der ökonomischen (H4) und der kulturellen Opportunitäten (H5) überprüft. In den Modellen 7 und 8 wird zunächst die Wohlstandsvariable eingeführt. Ein höherer Wohlstand hat einen statistisch signifikanten positiven Effekt auf den fairen Konsum und das Wissen über fair gehandelte Produkte. Ein höherer Wohlstand scheint somit eine Voraussetzung für den Kauf der etwas teureren ethischen Produkte zu sein. Dies bestätigt die Ergebnisse anderer Studien, die auf die Wichtigkeit von ökonomischen Opportunitätsstrukturen für politischen Konsum hinweisen (Koos 2011, 2012).

In den Modellen 9 und 10 (vgl. Tab. 3) wird die Wertewandelhypothese (H5) überprüft, also ob die ethischen Konsummuster mit der postmaterialistischen Kultur eines Landes zusammenhängen. Die Koeffizienten werden für beide abhängigen Variablen nicht signifikant. Die postmaterialistische Kultur scheint zu diesem Zeitpunkt also kein zentraler Treiber von Fair-Trade-Wissen und -Konsum zu sein. In den Modellen 11 und 12 wird für das Alter der organisierten Fair-Trade-Bewegung kontrolliert, um zu prüfen, ob die Diffusion des Fairen Handels durch einen früheren Gründungszeitpunkt begünstigt wird. Auch diese Variable hat keinen statistisch signifikanten Einfluss auf die beiden abhängigen Variablen.

Abschließend wird die Hypothese kontrolliert, ob die organisierte Vermarktlichung des Fairen Handels den Einfluss der sozialen Klasse auf den ethischen Konsum in Form eines distinktiven oder Statuskonsums erleichtert. Dazu werden in den Modellen 13 und 14 Cross-level-Interaktionen zwischen der organisierten Vermarktlichung und der Klassenvariable in die Regression eingeführt. Die statistisch signifikanten Effekte weisen darauf hin, dass die Vermarktlichung des Fairen Handels den ethischen Konsum als eine Möglichkeit der Statusdifferenzierung unterstützt. In Ländern mit einem aktiven Fair-Trade-Label weisen Personen in höheren Klassenpositionen eine ungleich größere Wahrscheinlichkeit für „distinktiven“ ethischen Konsum (verglichen mit der Referenzgruppe der unqualifizierten Arbeiter) auf, als in Ländern ohne eigenes Fair-Handels-Siegel. Diese Differenzierung tritt auch für die

\footnotetext{
${ }^{6}$ Die Ergebnisse der Untersuchung sind auf Anfrage erhältlich. Die mediale Resonanz wurde erfasst über eine Analyse von jeweils einer nationalen Tageszeitung im Jahr 1997 (z. B. El Pais in Spanien). Leider waren in der verwendeten Datenbank LexisNexis für diesen Zeitraum nur Zeitungen aus acht Ländern verfügbar. Es wurde nach Beiträgen mit dem allgemeinen Schlagwort Fair Trade bzw. Fairtrade, mit der jeweiligen nationalen Bezeichnung (z. B. Fairer Handel) und mit dem Namen des länderspezifischen Siegels (z. B. Transfair oder Max Havelaar) gesucht. Als Indikator wurde die Anzahl an Beiträgen für das Jahr ermittelt und sowohl anhand der Bevölkerungsgröße standardisiert als auch unstandardisiert in ein Regressionsmodell aufgenommen.
} 


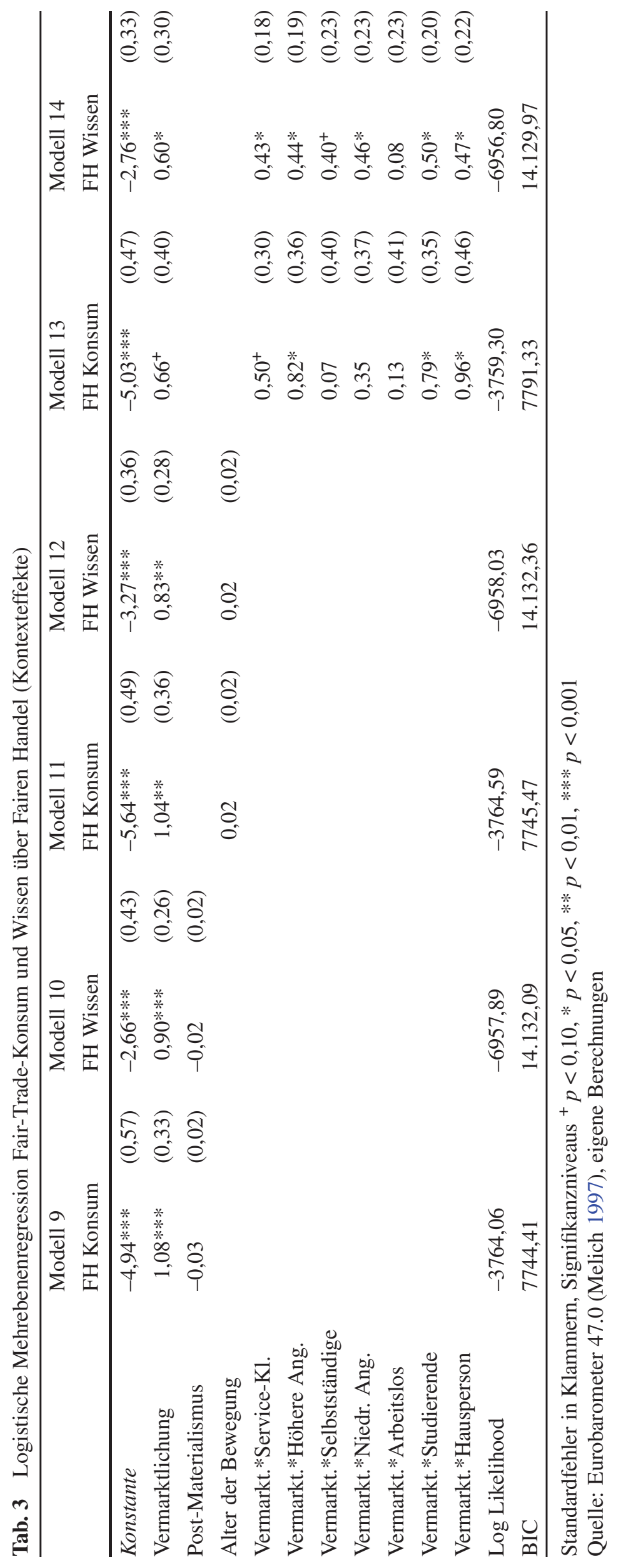


Wissensdimension zutage. Somit kann der distinktive Konsum als eine Triebfeder der Diffusion ethischen Konsums verstanden werden, der durch die organisationale Vermarktlichung einen wichtigen Impuls erhält.

\section{Der Anstieg des Fairen Handels im deutsch-britischen Vergleich}

Abschließend soll nun noch knapp anhand eines deutsch-britischen Vergleiches explorativ gezeigt werden, wie sich der ethische Konsum nach Einführung des FairTrade-Siegels seit 2001 entwickelt hat und wie der Anstieg dieses Konsumverhaltens erklärt werden kann. In Tab. 4 sind zu diesem Zweck nochmals die zentralen Erklärungsmechanismen anhand von vergleichenden Daten für Deutschland und Großbritannien über die Zeit abgetragen. Im direkten Vergleich zwischen dem Umsatz der Weltläden und des durch die gesiegelten Produkte erzielten Umsatzes wird deutlich, welchen starken Einfluss die neue Marktlogik im Vergleich zur traditionellen zivilgesellschaftlichen Logik gewonnen hat. Der Umsatz der Siegel-Produkte ist deutlich höher und steigt über die Zeit stärker an. Im Ländervergleich sieht man, dass bei einem fast ähnlichen Ausgangsniveau im Jahr 2001 der Umsatz von Fair-Trade-besiegelten Gütern in Großbritannien deutlich stärker gestiegen ist als in Deutschland. In beiden Länder wurden die Ausgaben für Kampagnen und Werbung mehr oder weniger kontinuierlich erhöht; der sprunghafte Anstieg beim Umsatz fair gehandelter Produkte kann folglich allein dadurch nicht erklärt werden. In Großbritannien sind die Ausgaben für diese Framing-Bestrebungen allerdings deutlich höher als in Deutschland, was eine mögliche Erklärung für die Länderunterschiede sein könnte. Die Anzahl an Verkaufsstellen bleibt in Großbritannien relativ konstant und deutlich unter dem Niveau von Deutschland, wo deren Anzahl langsam ansteigt. Aber auch hier kann die Zunahme an Verkaufsstellen den Umsatzsprung zwischen 2005 und 2007 nicht erklären.

Abschließend wird noch die Anzahl an Lizenznehmern, also der Anbieter von fair gehandelten Produkten betrachtet. Die Anzahl an Lizenznehmern ist in Großbritannien über die Zeit stark angestiegen. In Deutschland fällt dieser Anstieg vor allem mit dem Umsatzsprung zwischen den letzten beiden Zeitpunkten zusammen. Explorativ lässt sich dies als ein Hinweis darauf deuten, dass die Ausweitung an Anbietern

Tab. 4 Entwicklung des Fairen Handels in Deutschland und Großbritannien

\begin{tabular}{llrrrrr}
\hline & \multicolumn{2}{c}{ Deutschland } & \multicolumn{3}{c}{ Großbritannien } \\
& 2001 & 2005 & 2007 & 2001 & 2005 & 2007 \\
\hline Umsatz Weltläden (in Mio. $€$ ) & \multicolumn{1}{c}{ - } & 20,0 & 60,0 & 8,4 & 10,4 & 13,2 \\
Umsatz Label (in Mio. €) & 56,0 & 70,9 & 141,7 & 62,8 & 242,7 & 613,5 \\
Ausgaben für Werbung (in Mio. $€$ ) & 1,3 & 2,5 & 2,7 & 3,0 & 10,0 & 6,9 \\
Verkaufsstellen (in Tsd.) & 26,2 & 33,6 & 40,6 & 8,3 & 8,2 & 8,2 \\
Anzahl an Lizenznehmern & 85,0 & 87,0 & 100,0 & 39,0 & 178,0 & 252,0 \\
BIP pro Kopf (in KKP) & 30,2 & 31,4 & 33,4 & 32,6 & 32,7 & 34,1 \\
Postmaterialisten (in \%) & 10,2 & 11,6 & 12,9 & 12,9 & 15,0 & 10,6 \\
\hline
\end{tabular}

Quelle: siehe Datenbeschreibung 
einen möglichen Einfluss auf den Anstieg ethischen Konsums hat. In Großbritannien waren die Fair-Trade-Labelling-Organisationen nach 2001 sehr erfolgreich darin, neue Lizenznehmer zu akquirieren, und insbesondere Supermarktketten haben einen Teil ihrer Eigenmarken (z. B. für Schokoriegel) als Fair Trade zertifizieren lassen (Smith und Barrientos 2005). In Deutschland hat der Discounter Lidl im Jahr 2006 eine eigene Produktlinie mit Fair-Handels-Produkten eingeführt, was genau in den Zeitraum des starken Umsatzanstiegs hineinfällt. Die Anzahl und Art der Lizenznehmer scheinen also wichtige Impulsgeber für das Wachstum des ethischen Konsums zu sein. Neben der Produktdifferenzierung trägt auch der Wettbewerb zwischen den Anbietern um Kundensegmente zu einer weiteren Steigerung des Fairen Handels bei. Abschließend lässt sich festhalten, dass sich in den beiden Ländern weder das Pro-Kopf-Einkommen noch der Anteil der Postmaterialisten über die Zeit so verändert hat, dass dadurch der starke Anstieg des fairen Konsums erklärbar wäre. Die Länderunterschiede lassen sich auch kaum über die unterschiedlichen Opportunitätsstrukturen erklären.

\section{Fazit}

Die Fair-Trade-Bewegung will durch die Einführung bestimmter Produktionsstandards und die Durchsetzung fairer Preise die Bedingungen des Welthandels verändern. Sie verfolgt dabei das Ziel, sowohl den Umsatz des Fairen Handels zu steigern als auch die Menschen über die negativen Folgen der globalen Handelsbedingungen zu informieren. Ethischer Konsum ist ein integraler Bestandteil des Fairen Handels. Um die unterschiedliche Verbreitung des ethischen Konsums in Europa zu erklären, wurde in dem Beitrag in Ergänzung zu bestehenden akteurszentrierten Ansätzen versucht, der organisatorischen und strukturellen Einbettung der Konsumenten in unterschiedliche nationale Kontexte Rechnung zu tragen. Die Grundannahme lautete, dass die Fair-Handelsbewegung durch die Gründung von Fair-Trade-Labelling-Organisationen und die damit verbundene Besiegelung von Produkten entsprechend der Fair-Trade-Standards die Feldlogik der Bewegung hin zu einer organisationalen Vermarktlichung ethischen Tauschhandels verändert hat. Dies hat Auswirkungen auf die Verbreitung ethischen Konsums. Durch die Kommodifizierung normativer Standards mittels Produktsiegeln macht sich die Bewegung ökonomische Wettbewerbskräfte und statusinduzierte Differenzierungsstrategien der Konsumenten zunutze, um den Fairen Handel gesellschaftlich zu etablieren und den ethischen Konsum zu steigern. Dabei verliert die Fair-Trade-Bewegung keinesfalls ihren Charakter als soziale Bewegung. Die neue Feldlogik überlagert die bestehende zivilgesellschaftliche Logik, ohne diese jedoch vollständig zu verdrängen. Für einen frühen Zeitpunkt der Vermarktlichung des Fairen Handels im Jahr 1997 konnte gezeigt werden, wie die neue Feldlogik zur Entstehung des ethischen Konsums und zur Verbreitung des Wissens um den Fairen Handel beiträgt und welche Kontextfaktoren diese Entwicklung erklären können. Zu diesem Zeitpunkt sind weder die Kampagnenarbeit der Fair-Trade-Organisationen (Framing) noch die Verfügbarkeit von fair gehandelten Produkten (Mobilisierungsstrukturen) zentrale Faktoren für die Erklärung der damaligen Länderunterschiede im ethischen Konsum. 
Für den starken Anstieg des Fairen Handels nach Einführung des Siegels bedurfte es offensichtlich eines weiteren organisationalen Impulses, der über zwei Strategien in den Markt getragen wurde. Die Fair-Trade-Labelling-Organisationen nahmen zum einen die Konsumenten in den Blick und versuchten sie durch gezielte Kampagnenarbeit, Schulungen und Werbung auf ihre Anliegen aufmerksam zu machen. Zum anderen versuchten sie die Infrastruktur des Fairen Handels zu verbessern, indem Unternehmen als mögliche Lizenznehmer angesprochen und gewonnen wurden. Dieses „Klinken-Putzen“ bei den Unternehmen (so ein Vertreter von Transfair in einem Interview) war und ist eine wichtige Strategie, um die Verbreitung des ethischen Konsums voranzutreiben. Auch beim Vergleich der Wachstumstrends des Fairen Handels zwischen Deutschland und Großbritannien zeigt sich die Bedeutung der unternehmerischen Akzeptanz des Fair-Handels-Siegels und der damit einhergehenden Marktdifferenzierung. Die strategische Verwendung der Marktlogik durch eine soziale Bewegung kann also eine geeignete Möglichkeit sein, um die Interessen der Bewegung durchzusetzen. Die Fair-Handelsbewegung hat sich die Marktkräfte zu eigen gemacht, um den Markt selbst moralisch und sozial zu kolonisieren. Die organisierte Vermarktlichung ethischer Tauschbeziehungen führt auf diese Weise zu einer (partiellen) Moralisierung der Märkte.

Danksagung Für hilfreiche Kommentare und Anmerkungen zum Manuskript möchte ich mich bei den anonymen Gutachtern sowie Jörg Rössel und Henri Band herzlich bedanken.

\section{Literatur}

Arndorfer, V. A., \& Liebe, U. (2013). Consumer behavior in moral markets. On the relevance of identity, justice beliefs, social norms, status, and trust in ethical consumption. European Sociological Review, 29, 1251-1265.

Bartley, T., Koos, S., Samel, H., Setrini, G., \& Summers, N. (2015). Looking behind the label: Global industries and the conscientious consumer. Bloomington: Indiana University Press.

Benford, R. D., \& Snow, D. A. (2000). Framing processes and social movements: An overview and assessment. Annual Review of Sociology, 26, 611-639.

Bourdieu, P. (1984). La distinction: Critique sociale du jugement. Paris: Les Editions de Minuit.

Clemens, E. S. (1996). Organisational form as frame: Collective identity and political strategy in the american Labor Movement, 1880-1920. In D. McAdam, J.D. McCarthy \& M. N. Zald (Hrsg.), Comparative perspectives on social movements. Political opportunities, mobilizing structures and cultural framing (S. 205-226). Cambridge: Cambridge University Press.

Copeland, L. (2013). Value change and political action: Postmaterialism, political consumerism, and political participation. American Politics Research, 42, 257-282.

Davies, I. A. D. (2007). The eras and participants of fair trade: An industry structure/stakeholder perspective on the growth of the fair trade industry. Corporate Governance, 7, 455-470.

Davis, G. F., McAdam, D., Scott, R. W., \& Zald, M. N. (Hrsg.). (2005). Social movements and organisation theory. Cambridge: Cambridge University Press.

Ebbinghaus, B., \& Visser, J. (1999). When institutions matter: Union growth and decline in Western Europe, 1950-1995. European Sociological Review, 15, 135-158.

Elster, J. (1989). Nuts and bolts for the social sciences. Cambridge: Cambridge University Press.

Erikson, R., \& Goldthorpe, J.H. (1992). The constant flux: A study of class mobility in industrial societies. Oxford: Clarendon Press.

Ertman, M.M., \& Williams, J.C. (2005). Preface. Freedom, equality, and the many futures of commodification. In M. M. Ertman \& J. C. Williams (Hrsg.), Rethinking commodification (S. 1-7). New York: New York University Press. 
Eshuis, F., \& Harmsen, J. (2003). Making trade work for the producers: 15 years of Fairtrade labelled coffee in the Netherlands. Max Havelaar Foundation, Netherlands. http://s3.amazonaws.com/zanran_ storage/www.fairtrade.org.uk/ContentPages/51254453.pdf. Zugegriffen: Aug. 2016.

Eurostat (2009). Annual detailed enterprise statistics on trade. http://epp.eurostat.ec.europa.eu/portal/page/ portal/eurostat/home/. Zugegriffen: Jan. 2010.

Feddersen, T. J., \& Gilligan, T.W. (2001). Saints and markets: Activists and the supply of credence goods. Journal of Economics \& Management Strategy, 10, 149-171.

FLO (2005). Annual Report 2003/04. Bonn: Fairtrade Labelling Organization International.

FLO (2006). Annual Report 2004/05. Bonn: Fairtrade Labelling Organization International.

FLO (2008). Annual Report 2006/07. Bonn: Fairtrade Labelling Organization International.

FLO (2010). Growing stronger together. Annual Report 2009-10. Bonn: Fair Trade Labelling Organizations International.

Fridell, G. (2004). The Fair Trade network in historical perspective. Candian Journal of Development Studies, 25, 411-428.

Fridell, G. (2007). Fair-Trade coffee and commodity fetishism: The limits of market-driven social justice. Historical Materialism, 15, 79-104.

Gamson, W. A., \& Meyer, D.S. (1996). Framing political opportunity. In D. McAdam, J.D. McCarthy \& M.N. Zald (Hrsg.), Comparative perspectives on social movements. Political opportunities, mobilizing structures and cultural framing (S. 275-290). Cambridge: Cambridge University Press.

Goffman, E. (1975). Frame analysis: An essay on the organization of the experience. Harmondsworth: Penguin Books.

Guo, G., \& Zhao, H. (2000). Multilevel modeling for binary data. Annual Review of Sociology, 26, $441-462$.

Hagenaars, A. J. M., de Vos, K., \& Zaidi, A. (1994). Poverty statistics in the late 1980s: Research based on micro-data. Luxemburg: Office for Official Publications of the European Communities.

Hechter, M. (1994). The role of values in rational choice theory. Rationality and Society, 6, 318-333.

Hox, J. (2002). Multilevel analysis. Techniques and applications. New Jersey: Lawrence Erlbaum Associates.

Inglehart, R. (1997). Modernization and postmodernization: Cultural, economic, and political change in 43 societies. Princeton: Princeton University Press.

Koos, S. (2011). Varieties of environmental labelling, market structures, and sustainable consumption across Europe: A comparative analysis of organizational and market supply determinants of environmental-labelled goods. Journal of Consumer Policy, 34, 127-151.

Koos, S. (2012). What drives political consumption in Europe? A multi-level analysis on individual characteristics, opportunity structures and globalization. Acta Sociologica, 55, 37-57.

Krier, J. M. (2001). Fair Trade in Europe 2001. Facts and figures on the Fair Trade sector in 18 European countries. Maastricht: European Fair Trade Organization.

Krier, J. M. (2006). Fair Trade in Europe 2005. Facts and figures on the Fair Trade in 25 European countries. Maastricht: European Fair Trade Organization.

Krier, J. M. (2007). Fair Trade 2007: New facts and figures from an ongoing success story. A report on Fair Trade in 33 consumer countries. Culemborg: Dutch Association of Worldshops.

Linton, A., Liou, C. C., \& Shaw, K. A. (2004). A taste of trade justice: Marketing global social responsibility via Fair Trade coffee. Globalizations, 1, 223-246.

Lounsbury, M. (1997). Exploring the institutional toolkit. The rise of recycling in the US solid waste field. American Behavioural Scientist, 49, 465-477.

Lounsbury, M., Ventresca, M., \& Hirsch, P. M. (2003). Social movements, field frames, and industry emergence: A cultural-political perspective on US recycling. Socio-Economic Review, 1, 71-104.

Martinelli, E. (1995). Fair Trade in Europe. Facts and figures on the Fair Trade sector in 14 European countries. Maastricht: European Fair Trade Association.

Martinelli, E. (1998). Fair Trade in Europe. Facts and figures on the Fair Trade sector in 16 European countries. Maastricht: European Fair Trade Association.

Martín-Lagos López, M.D. (2010). Consumption and modernization in the European Union. European Sociological Review, 27, 124-137.

McAdam, D. (1996). Conceptual origins, current problems, future directions. In D. McAdam, J. D. McCarthy \& M. N. Zald (Hrsg.), Comparative perspectives on social movements. Political opportunities, mobilizing structures and cultural framing (S. 23-40). Cambridge: Cambridge University Press.

McAdam, D., \& Scott, R. W. (2005). Organizations and movements. In G. F. Davis, D. McAdam, R. W. Scott \& M. N. Zald (Hrsg.), Social movements and organisation theory (S. 4-40). Cambridge: Cambridge University Press. 
McAdam, D., McCarthy, J.D., \& Zald, M.N. (1996a). Introduction: Opportunities, mobilizing structures, and framing processes - Towards a synthetic, comparative perspective on social movements. In D. McAdam, J.D. McCarthy \& M.N. Zald (Hrsg.), Comparative perspectives on social movements. Political opportunities, mobilizing structures and cultural framing (S. 1-20). Cambridge: Cambridge University Press.

McAdam, D., McCarthy, J.D., \& Zald, M.N. (Hrsg.). (1996b). Comparative perspectives on social movements. Political opportunities, mobilizing structures and cultural framing. Cambridge: Cambridge University Press.

McCarthy, J.D. (1996). Constraints and opportunities in adopting, adapting, and inventing. In D. McAdam, J.D. McCarthy \& M.N. Zald (Hrsg.), Comparative perspectives on social movements. Political opportunities, mobilizing structures and cultural framing (S. 141-151). Cambridge: Cambridge University Press.

McCarthy, J.D., \& Zald, M.N. (1977). Resource mobilization and social movements: A partial theory. American Journal of Sociology, 82, 1212-1241.

Melich, A. (1997). Eurobarometer 47.0: Images of Germany, consumer issues, electronic information, and Fair Trade practices. Cologne: Zentralarchiv für Empirische Sozialforschung.

Meyer, D. S. (2004). Protest and political opportunities. Annual Review of Sociology, 30, 125-145.

Meyer, J.W. (2010). World society, institutional theories and the actor. Annual Review of Sociology, 36, $1-20$.

Micheletti, M. (2003). Political virtue and shopping. Individuals, consumerism, and collective action. New York: Palgrave Macmillan.

Micheletti, M. (2004). Why more women? Issues of gender and political consumption. In M. Micheletti, A. Follesdal \& D. Stolle (Hrsg.), Politics, products, and markets (S. 245-264). New Brunswick London: Transaction Publishers.

Micheletti, M., \& Stolle, D. (2012). Sustainable citizenship and the new politics of consumption. The ANNALS of the American Academy of Political and Social Science, 644, 88-120.

Neilson, L. A., \& Paxton, P. (2010). Social capital and political consumerism: A multilevel analysis. Social Problems, 57, 5-24.

Nicholls, A., \& Opal, C. (2005). Fair Trade: Market-driven ethical consumption. London: Sage Publications.

Opp, K.-D. (1999). Contending conceptions of the theory of rational action. Journal of Theoretical Politics, $11,171-202$.

Pelsmacker, P. de, \& Janssens, W. (2007). A model for Fair Trade buying behaviour: The role of perceived quantity and quality of information and of product-specific attitudes. Journal of Business Ethics, 75, 361-380.

Pfeiffer, M. (2009). Public opinion on state responsibility for minimum income protection: A comparison of 14 European countries. Acta Sociologica, 52, 117-134.

Rao, H. (1998). Caveat emptor: The construction of nonprofit consumer watchdog organizations. American Journal of Sociology, 130, 912-961.

Rao, H., Morrill, C., \& Zald, M.N. (2000). Power plays: How social movements and collective action create new organizational forms. Research in Organizational Behaviour, 22, 239-282.

Renard, M.-C. (2003). Fair Trade: Quality, market and conventions. Journal of Rural Studies, 19, 87-96.

Rose, D., \& Harris, E. (2007). The European socio-economic classification: A new social class schema for comparative European research. European Societies, 9, 459-490.

Rössel, J. (2008). Conditions for the explanatory power of life styles. European Sociological Review, 24, 231-241.

Scott, W. R. (2008). Institutions and organisations. Ideas and Interests. Thousand Oaks, CA: SAGE.

Scott, W. R., Ruef, M., Mendel, P. J., \& Caronna, C. A. (2000). Institutional change and healthcare organizations: From professional dominace to managed care. Chicago: Chicago University Press.

Shaw, D., Grehan, E., Shiu, E., Hassan, L., \& Thomson, J. (2005). An exploration of values in ethical consumer decision making. Journal of Consumer Behaviour, 4, 185-200.

Smith, S., \& Barrientos, S. (2005). Fair Trade and ethical trade: Are there moves towards convergence? Sustainable Development, 13, 190-198.

Snow, D. A., \& Benford, R.D. (1988). Ideology, frame ressonance, and participant mobilization. International Social Movement Research, 1, 197-217.

Southerton, D., Chappells, H., \& Van Vliet, B. (Hrsg.). (2004). Sustainable consumption: The implications of changing infrastructures of provision. Cheltenham: Edward Elgar.

Stehr, N. (2007). Die Moralisierung der Märkte. Eine Gesellschaftstheorie. Frankfurt a.M.: Suhrkamp. 
Stolle, D., \& Hooghe, M. (2004). Consumers as political participants? Shifts in political action repertoires in western societies. In M. Micheletti, A. Follesdal \& D. Stolle (Hrsg.), Politics, products, and markets (S. 265-288). New Brunswick London: Transaction Publisher.

Stolle, D., Hooghe, M., \& Micheletti, M. (2005). Politics in the supermarket: Political consumerism as a form of political participation. International Political Science Review, 26, 245-269.

Strømsnes, K. (2005). Political consumption in Norway: Who, why - and does it have any effect? In M. Boström, A. Føllesdal, M. Klintman, M. Micheletti \& M. P. Sørensen (Hrsg.), Political consumerism: Its motivations, power, and conditions in the Nordic countries and elsewhere (S. 165-181). Copenhagen: Nordic Council of Ministers.

Sunderer, G., \& Rössel, J. (2012). Morality or economic interest? The impact of moral motives and economic factors on the purchase of Fair Traded groceries. International Journal of Consumer Studies, 36, 244-250.

Tarrow, S. (1994). Power in movement: Social movements, collective action and politics. Cambridge: Cambridge University Press.

Thelen, K. (2004). How institutions evolve: The political economy of skills in Germany, Britain, the United States, and Japan. Cambridge: Cambridge University Press.

Thornton, P. H., \& Ocasio, W. (2008). Institutional logics. In R. Greenwood, C. Oliver, K. Sahlin-Andersson \& R. Suddaby (Hrsg.), The SAGE Handbook of organizational institutionalism. Thousand Oaks, CA: SAGE.

Verba, S. (1965). Comparative political culture. In L. W. Pye \& S. Verba (Hrsg.), Political culture and political development (S. 512-560). Princeton: Princeton University Press.

Verba, S., Schlozman, K., \& Brady, H.E. (1995). Voice and equality: Civic voluntarism in American politics. Cambridge: Harvard University Press.

Wahlström, M., \& Peterson, A. (2006). Between the state and the market: Expanding the concept of ,political opportunity structure“. Acta Sociologica, 49, 363-377.

Sebastian Koos geb. 1978. Juniorprofessor für Corporate Social Responsibility am Fachbereich für Politik- und Verwaltungswissenschaften der Universität Konstanz. Promotion in Soziologie zum Thema „Consumer and Corporate Responsibility across Europe“ an der Universität Mannheim. 2015 bis 2016 John F. Kennedy Memorial Fellow am Center for European Studies der Universität Harvard, USA. Forschungsschwerpunkte: Nachhaltiger und politischer Konsum, gesellschaftliche Verantwortung von Unternehmen, industrielle Beziehungen. Ausgewählte Veröffentlichung: (mit T. Bartley, H. Samel, G. Setrini und N. Summers) Looking behind the label. Global industries and the conscientious consumer, 2015. 